\title{
Adaptive Fuzzy Synchronization of Fractional-Order Chaotic (Hyperchaotic) Systems with Input Saturation and Unknown Parameters
}

\author{
Heng Liu, ${ }^{1}$ Ye Chen, ${ }^{2}$ Guanjun Li, ${ }^{1}$ Wei Xiang, ${ }^{1}$ and Guangkui Xu ${ }^{1}$ \\ ${ }^{1}$ Department of Applied Mathematics, Huainan Normal University, Huainan 232038, China \\ ${ }^{2}$ College of Mathematics and Information Science, Shaanxi Normal University, Xian 710119, China
}

Correspondence should be addressed to Heng Liu; liuheng122@gmail.com

Received 20 July 2017; Accepted 4 October 2017; Published 2 November 2017

Academic Editor: Abdelalim Elsadany

Copyright (c) 2017 Heng Liu et al. This is an open access article distributed under the Creative Commons Attribution License, which permits unrestricted use, distribution, and reproduction in any medium, provided the original work is properly cited.

\begin{abstract}
We investigate the synchronization problem of fractional-order chaotic systems with input saturation and unknown external disturbance by means of adaptive fuzzy control. An adaptive controller, accompanied with fractional adaptation law, is established, fuzzy logic systems are used to approximate the unknown nonlinear functions, and the fractional Lyapunov stability theorem is used to analyze the stability. This control method can realize the synchronization of two fractional-order chaotic or hyperchaotic systems and the synchronization error tends to zero asymptotically. Finally, we show the effectiveness of the proposed method by two simulation examples.
\end{abstract}

\section{Introduction}

Recent studies showed that a large number of physical phenomena of nature and chemical processes, such as viscosity systems, colored noise, electrolyte electrode polarization, electromagnetic waves, and many actual systems can be described by fractional-order differential equations, making the slowly developed fractional calculus be a resurgence of interest [1-10]. Today, fractional-order systems described by fractional operators play a very important role in control fields [11-13]. Using the traditional integer-order differential equations as a method of describing dynamic system models has great limitation in biological engineering, cell engineering, neural network engineering, and some other emerging fields. However, the models established by fractional calculus can often achieve more satisfying and unexpected results [9, $14,15]$. Actually, the physical models established by the theory of fractional-order calculus are more concise and accurate in presentation when describing the complex problems of physics. In addition, the fractional controller not only can expand the freedom of the controlled system but also is able to obtain better control performance. Furthermore, the feature that fractional calculus has the function of memory makes the system's states in the future be related to the previous and current states. Thus the memory and genetic characteristics of certain processes and materials can be expressed more accurately, which is conducive to improving the control effect of the systems $[16,17]$.

It is well known that chaos has potential application values and great prospect in secure communication and other areas [18-22]. Recently, fractional-order chaotic systems and hyperchaotic systems have been studied in a widespread way and have been payed close attention with the deepening of theoretical research of fractional-order systems [23-26]. Many scholars have studied the synchronization control problems for fractional-order chaotic systems. So far, there are many control methods for fractional-order nonlinear chaotic systems (such as drive-response method, finite-time synchronization, nonlinear feedback method, adaptive synchronization control method, nonlinear disturbance observer method, nonlinear coupling method, sliding method, PC method, Lyapunov function activated method, 
and synchronization control method [27-33]). It is worth noting that the above literatures which study the problem of fractional-order chaotic systems synchronization have a basic assumption that the controller does not have any restrictions. However, almost all actuators in actual control systems have full amplitude or amplitude constraint problem (the amplitude of the output of the controller is limited artificially for reliability [34]). In addition, the presence of the input saturation of the control systems tends to attenuate the good performance of the system and even leads to instability of the closed-loop system. So many scholars have conducted extensive research in integer-order systems with input saturation in the recent years (literatures [35-37], etc.). Little work has been done to study the synchronous control of fractional-order nonlinear chaotic systems with input saturation.

In this paper, the adaptive fuzzy synchronization of uncertain fractional-order nonlinear systems with input saturation and external disturbance is investigated on the basis of the above discussions. Fuzzy logic systems are used to approximate the fully unknown nonlinear functions of the systems. A fractional adaptive fuzzy synchronization controller is designed, and we prove the stability of the chaotic systems according to the fractional Lyapunov stability criterion. The main work of this paper can be concluded as follows: (1) The synchronization of fractional-order chaotic systems with input saturation and external disturbance is discussed in this paper. (2) An adaptive fuzzy synchronization controller is designed and fractional adaptive laws are designed to update the values of the parameters online.

\section{Preliminaries}

2.1. Preliminaries of Fractional Calculus. With the history of more than 300 years, there are many definitions of fractional calculus. But the most commonly used definitions are Grünwald-Letnikov, Caputo, and Riemann-Liouville definitions [11]. We choose Caputo's derivative in this paper as its Laplace transform requires the initial values of the classical integer-order systems.

The $\alpha$ th fractional integral operator is defined as

$$
\mathscr{I}_{\tau}^{-\alpha} f(\tau)={ }_{0} D_{\tau}^{-\alpha} f(\tau)=\frac{1}{\Gamma(\alpha)} \int_{0}^{\tau}(\tau-\nu)^{\alpha-1} f(\nu) d \nu
$$

where $\Gamma(z)=\int_{0}^{\infty} \tau^{z-1} e^{-\tau} d \tau$ represents Euler's Gamma function. The $\alpha$ th fractional derivative operator is given as

$$
D_{t}^{\alpha} f(\tau)=\frac{1}{\Gamma(n-\alpha)} \int_{0}^{\tau}(\tau-\nu)^{n-\alpha-1} f^{(n)}(\nu) d \nu,
$$

where $n-1 \leq \alpha<n$ and $n$ is an integer. And the Laplace transform of the formed formula (2) is

$$
\int_{0}^{\infty} e^{-s t} D_{\tau}^{\alpha} f(\tau) d \tau=s^{\alpha} F(s)-\sum_{k=0}^{n-1} s^{\alpha-k-1} f^{(k)}(0) .
$$

The following properties of fractional calculus hold.
Property 1 (see [38]). Suppose that $x(\tau) \in C^{1}[0, T], T>0$; then

$$
D_{\tau}^{\alpha_{1}} D_{\tau}^{\alpha_{2}} x(\tau)=D_{\tau}^{\alpha_{1}+\alpha_{2}} x(\tau)
$$

where $\alpha_{1}, \alpha_{2}>0$ and $\alpha_{1}+\alpha_{2} \leqslant 1$.

Property 2 (see [38]). The linearity of the Caputo fractional operator is as follows:

$$
D_{\tau}^{\alpha}(\lambda x(\tau)+\omega y(\tau))=\lambda D_{\tau}^{\alpha} x(\tau)+\omega D_{\tau}^{\alpha} y(\tau),
$$

where $\lambda$ and $\omega$ are two real constants.

Remark 1. If $c$ is a constant, then its Caputo derivative is 0 . Namely, $D_{\tau}^{\alpha} c=0$. Particularly, we have $D_{\tau}^{\alpha} 0=0$.

Property 3 (see [11]). Let $x(\tau) \in C^{1}[0, T]$ and $0<\alpha<1$; then

$$
\begin{aligned}
& D_{\tau}^{-\alpha} D_{\tau}^{\alpha} x(\tau)=x(\tau)-x(0), \\
& D_{\tau}^{\alpha} D_{\tau}^{-\alpha} x(\tau)=x(\tau)
\end{aligned}
$$

Note that the above properties hold if and only if $0<\alpha<$ 1. Consequently, only the case where $0<\alpha<1$ is involved in the controller design and stability analysis. For convenience, in the rest of this paper, we always assume that $\alpha \in(0,1)$.

2.2. Fuzzy Logic Systems. A fuzzy logic system (FLS) consists of four parts (cf. [8, 9, 39-44]): the knowledge base, the fuzzifier, the fuzzy inference engine working on the fuzzy rules, and the defuzzifier. Usually, a fuzzy logic system is modeled by

$$
\widehat{y}=\frac{\sum_{j \in J} w_{j}(t) \mu_{j}(x(t))}{\sum_{j \in J} \mu_{j}(x(t))}
$$

where $\hat{y}$ (a Lipschitz continuous mapping from a compact subset $\Omega \subseteq \mathscr{R}^{n}$ to the real line $\mathscr{R}$ ) is called the output of the fuzzy logic system, $x=\left[x_{1}, \ldots, x_{n}\right]^{T} \in C^{1}[\mathscr{I}, \Omega]$ (the set of all continuous mappings from $\mathscr{I}=[0,+\infty) \subseteq \mathscr{R}$ to $\Omega$ which have continuous derivatives) is called the input vector which is defined by $x(t)=\left[x_{1}(t), \ldots, x_{n}(t)\right]^{T}(\forall t \in \mathscr{I})$, $J=\prod_{i=1}^{n} \mathscr{F}_{i}, \mathscr{F}_{i}$ consists of $N_{i}$ fuzzy sets $(1 \leq i \leq n), \mu_{j}$ (a mapping from $\mathscr{R}^{n}$ to the closed unit interval $[0,1] \subseteq \mathscr{R}$ ) is called the membership function of rule $j(j \in J)$, and $w_{j}$ (a mapping from $\mathscr{I}$ to $\mathscr{R}$ ) is called the centroid of the $j$ th consequent set $(j \in J)$; we may identify $J$ with $\{1,2, \ldots, N\}$ for the sake of convenience. Write $\mathscr{W}(t)=\left[w_{1}(t), \ldots, w_{N}(t)\right]^{T}$ and $\varphi(x(t))=\left[q_{1}(x(t)), q_{2}(x(t)), \ldots, q_{N}(x(t))\right]^{T}$, where $q_{j}$ (called the $j$ th fuzzy basis function, $j \in J$ ) is a continuous mapping (and thus $\varphi \Omega \rightarrow \mathscr{R}^{N}$ is continuous) defined by

$$
q_{j}(x(t))=\frac{w_{j}(t)}{\sum_{s \in J} \mu_{s}(x(t))} .
$$

Then system (7) can be rewritten as

$$
y=\mathscr{W}^{T} \varphi(X)
$$


In contrast to conventional control techniques, fuzzy logic systems are best utilized in complex ill-defined processes that can be controlled by a skilled human operator without much knowledge of their underlying dynamics. The basic idea behind fuzzy logic systems is to incorporate the "expert experience" of a human operator in the design of the controller in controlling a process whose input-output relationship is described by a collection of fuzzy control rules involving linguistic variables rather than a complicated dynamic model.

The fuzzy logic system (9) is employed to approximate the unknown nonlinear function $f(\mu)$ in this paper. It can be expressed as

$$
f(\mu)=\mathscr{W}^{* T} \varphi(\mu)+\epsilon(\mu),
$$

where $\epsilon(\mu)$ is the ideal vector of the approximation error. $\mathscr{W}^{*}$ is the ideal weight matrix which can be expressed as

$$
\mathscr{W}^{*}=\arg \min _{\mathscr{W}}[\sup |\hat{f}(\mu)-f(\mu)|],
$$

where $\hat{f}(\mu)$ is the estimation of $f(\mu)$.

\section{Adaptive Fuzzy Synchronization Controller Design and Stability Analysis}

3.1. Problem Statement. Consider the following fractionalorder chaotic systems:

$$
\begin{aligned}
& D_{t}^{\alpha} x(t)=A x(t)+f(x(t)), \\
& D_{t}^{\alpha} y(t)=C y(t)+g(y(t))+\operatorname{sat}(u)+d(t),
\end{aligned}
$$

where $x(t)=\left[x_{1}(t), \ldots, x_{n}(t)\right]^{T} \in R^{n}$ is the state vector of the drive system (12) and $y(t)=\left[y_{1}(t), \ldots, y_{n}(t)\right]^{T} \in R^{n}$ is the state vector of the response system (13). $f_{i}(\cdot)$ and $g_{i}(\cdot)$, $i=1,2, \ldots, n$, are two unknown nonlinear functions, $A$ and $C \in R^{n \times n}$ are two constant matrices, $d(t) \in R^{n}$ is the external disturbance, $\operatorname{sat}(u) \in R^{n}$ represents the input saturation, and $u(t) \in R^{n}$ is the control input.

Remark 2. In theoretical analysis, one often hopes that the input value and the output value can keep proportionally synchronized change when the former is relatively small. However, when the input value increases to a certain extent due to the system limitation factor, the output value of the actual conditions is no longer increasing but tends to or stays at a certain value in practical systems. This is said to be "saturation" phenomenon which is shown in Figure 1.

Definition 3 (see $[45,46])$. A mapping from $R^{n}$ to $R^{n}$ sat : $v \rightarrow \operatorname{sat}(v)$ is called a saturator, where $v=\left[v_{1}, v_{2}, \ldots, v_{n}\right]^{T} \in$ $R^{n}$ and $\operatorname{sat}(v)=\left[\operatorname{sat}\left(v_{1}\right), \operatorname{sat}\left(v_{2}\right), \ldots, \operatorname{sat}\left(v_{n}\right)\right]^{T}$. The definition of sat $(u)$ is as follows:

$$
\operatorname{sat}(u(t))= \begin{cases}u_{r}, & u(t) \geq u_{r} \\ u(t), & u_{l}<u(t)<u_{r} \\ u_{l}, & u(t) \leqslant u_{l} .\end{cases}
$$

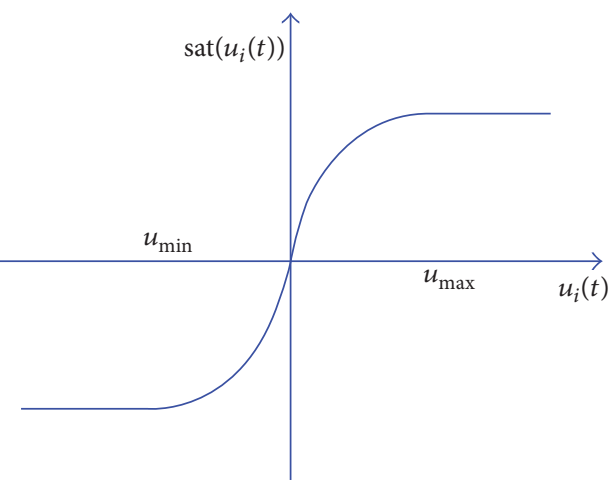

Figure 1: The phenomenon of saturation.

Suppose that the part that exceeds the saturation limiter is referred to as $\delta(t)$; then one has

$$
\delta(t)= \begin{cases}u_{r}-u(t), & u(t) \geq u_{r} \\ 0, & u_{l}<u(t)<u_{r} \\ u_{l}-u(t), & u(t) \leqslant u_{l},\end{cases}
$$

where $u_{r}$ and $u_{l}$ are called saturated amplitude satisfying $u_{l}<$ 0 and $u_{r}>0$.

Assumption 4. The external disturbance $d_{i}(\cdot)$ is a bounded continuous function. Namely, there exists an unknown constant $\rho_{i}>0$ such that

$$
\left|d_{i}(\cdot)\right| \leqslant \rho_{i}, \quad(i=1,2, \ldots, n) .
$$

Remark 5. It should be pointed out that Assumption 4 is reasonable. We just need the boundaries of the external disturbances, and their exact values are not needed in the process of designing the controller.

The objective of our work is to design an appropriate adaptive fuzzy controller such that the synchronization error $e(t)=y(t)-x(t)$ tends to zero asymptotically (namely, $\left.\lim _{t \rightarrow \infty}\|e(t)\|=0\right)$.

3.2. Controller Design. The dynamical equation of the synchronization error can be described as

$$
\begin{aligned}
D_{t}^{\alpha} e(t)= & C e(t)+(C-A) x(t)+g(y(t))-f(x(t)) \\
& +\operatorname{sat}(u)+d(t) .
\end{aligned}
$$

Based on the definition of $\operatorname{sat}(u)$, we can obtain that

$$
\operatorname{sat}(u)=u(t)+\delta(t)
$$

Then (17) can be rewritten as

$$
\begin{aligned}
D_{t}^{\alpha} e(t)= & C e(t)+(C-A) x(t)+g(y(t))-f(x(t)) \\
& +u(t)+\delta(t)+d(t) .
\end{aligned}
$$


Consider

$$
\begin{aligned}
h(x(t), y(t))= & h(t) \\
= & (C-A) x(t)+g(y(t))-f(x(t)) \\
& +\delta(t) .
\end{aligned}
$$

Nothing that the nonlinear function $h(t)$ is unknown, it can be approximated, through the fuzzy logic system (9), as

$$
\widehat{h}_{i}(t)=\mathscr{W}_{i}^{T}(t) \varphi_{i}(x),
$$

where $\mathscr{W}_{i}^{*}=\arg \min _{\mathscr{W}_{i}(t)}\left[\sup \left|h_{i}(t)-\widehat{h}_{i}\left(t, \mathscr{W}_{i}(t)\right)\right|\right]$. Let the unknown constant estimation error of the fuzzy logic systems and the approximation error, respectively, be

$$
\begin{aligned}
\widetilde{\mathscr{W}}_{i}(t) & =\mathscr{W}_{i}(t)-\mathscr{W}_{i}^{*}, \\
\epsilon_{i}(t) & =h_{i}(t)-\widehat{h}_{i}\left(t, \mathscr{W}_{i}^{*}\right) .
\end{aligned}
$$
design.

The following assumption is needed in the controller

Assumption 6. Suppose that the estimation error $\epsilon_{i}(t)$ is bounded; namely, $\left|\epsilon_{i}\right| \leqslant \epsilon_{i}{ }^{*}$, where $\epsilon_{i}{ }^{*}>0$ is an unknown constant $(i=1,2, \ldots, n)$.

Then the estimated error of unknown nonlinear function $h(t)$ can be written as

$$
\begin{aligned}
\widehat{h}(t, \mathscr{W})-h(t)= & \widehat{h}(t, \mathscr{W})-\widehat{h}\left(t, \mathscr{W}^{*}\right)+\widehat{h}\left(t, \mathscr{W}^{*}\right) \\
& -h(t) \\
= & \widehat{h}(t, \mathscr{W})-\widehat{h}\left(t, \mathscr{W}^{*}\right)-\epsilon(t) \\
= & \widetilde{\mathscr{W}}^{T}(t) \varphi(x)-\epsilon(t),
\end{aligned}
$$

where $\mathscr{W}^{*}=\left[\mathscr{W}_{1}^{*}, \mathscr{W}_{2}^{*}, \ldots, \mathscr{W}_{m}^{*}\right]^{T}$.

Based on the above discussion, the synchronization controller $u(t)$ can be designed as

$$
\begin{aligned}
u(t)= & -K e(t)-\mathscr{W}^{T}(t) \varphi(x)-H \operatorname{sign}(e(t)) \\
& -\hat{\rho} \operatorname{sign}(e(t))
\end{aligned}
$$

where $K=\operatorname{diag}\left[k_{1}, k_{2}, \ldots, k_{n}\right] \in R^{n \times n}$ and $k_{i}>0$ is the designed parameter. $H=\operatorname{diag}\left[\epsilon_{1}^{*}, \ldots, \epsilon_{n}^{*}\right], \hat{\rho}=$ $\operatorname{diag}\left[\hat{\rho}_{1}, \widehat{\rho}_{2}, \ldots, \hat{\rho}_{n}\right] \in R^{n \times n}$, and $\hat{\rho}_{i}$ is the estimation of $\rho_{i}(i=$ $1,2, \ldots, n)$. The fuzzy parameters and $\hat{\rho}_{i}(t)$ are, respectively, updated by

$$
\begin{aligned}
D_{t}^{\alpha} \mathscr{W}_{i}(t) & =\mu_{i} e_{i}(t) \varphi_{i}(x), \\
D_{t}^{\alpha} \widehat{\rho}_{i}(t) & =\gamma_{i}\left|e_{i}(t)\right|,
\end{aligned}
$$

where $\mu_{i}, \gamma_{i}>0$ are positive design parameters.

Remark 7. The above fractional adaptive laws are used to update the adjustable parameters. Notice that (26) can also be written as the following equation:

$$
\widehat{\rho}_{i}(\nu)=\widehat{\rho}_{i}(0)+\frac{1}{\Gamma(\alpha)} \int_{0}^{\nu} \gamma_{i}(\nu-\tau)^{\alpha-1}\left|e_{i}(\tau)\right| d \tau .
$$

Definition 8 (see [11]). Mittag-Leffler functions (M-L functions) with one parameter and two parameters are, respectively, defined as

$$
\begin{gathered}
E_{\alpha}(t)=\sum_{k=0}^{\infty} \frac{t^{k}}{\Gamma(\alpha k+1)}, \\
E_{\alpha, \beta}(t)=\sum_{k=0}^{\infty} \frac{t^{k}}{\Gamma(\alpha k+\beta)},
\end{gathered}
$$

where $\alpha, \beta>0$. The Laplace transform of (29) is expressed as

$$
\mathscr{L}\left(t^{\beta-1} E_{\alpha, \beta}\left(-\lambda t^{\alpha}\right)\right)=\frac{s^{\alpha-\beta}}{s^{\alpha}+\lambda}, \quad\left(\operatorname{Re}(s)>|\lambda|^{1 / \alpha}\right),
$$

where $t \geq 0$ and $\lambda \in R$.

Lemma 9 (fractional Lyapunov second method [11]). Let the origin be the equilibrium point of the following system:

$$
D_{t}^{\alpha} x(t)=f(t, x(t))
$$

where $x(t) \in R^{n}$ is the system variable and $f(t, x(t)) \in$ $R^{n}$ is nonlinear function that satisfies the local Lipschitz condition. If there exists Lyapunov function $\mathscr{V}(t, x(t))$ and positive parameters $h_{1}, h_{2}$, and $h_{3}$ such that

$$
\begin{aligned}
h_{1}\|x(t)\| & \leq \mathscr{V}(t, x(t)) \leq h_{2}\|x(t)\|, \\
D_{t}^{\alpha} \mathscr{V}(t, x(t)) & \leq-h_{3}\|x(t)\|,
\end{aligned}
$$

then system (31) is asymptotically stable.

Lemma 10 (see [47]). Suppose that $x(t) \in R^{n}$ is a continuously differentiable function; then one has

$$
\frac{1}{2} D_{t}^{\alpha} x^{T}(t) x(t) \leq x^{T}(t) D_{t}^{\alpha} x(t) .
$$

Lemma 11 (fractional monotonic principle [47]). If $D_{t}^{\alpha} x(t) \geq$ 0 , then $x(t)$ is monotonically increasing in $[0,+\infty)$. If $D_{t}^{\alpha} x(t) \leqslant$ 0 , then $x(t)$ is monotonically decreasing in $[0,+\infty]$.

Lemma 12 (see $[9,47])$. Let $V_{1}(t)=(1 / 2) x^{2}(t)+(1 / 2) y^{2}(t)$; $x(t)$ and $y(t) \in R$ are two continuous functions. If there exists a positive constant $k$ satisfying

$$
D_{t}^{\alpha} V_{1}(t) \leqslant-k x^{2}(t)
$$

then one has the following inequality:

$$
x^{2}(t) \leqslant 2 V_{1}(0) E_{\alpha}\left(-2 k t^{\alpha}\right) .
$$

Lemma 13. Suppose that $V(t)=(1 / 2) x^{T}(t) x(t)+$ $(1 / 2) y^{T}(t) y(t)$, where $x(t), y(t) \in R^{n}$ have continuous derivative. If there exists a constant $h>0$ such that

$$
D_{t}^{\alpha} V(t) \leqslant-h x^{T}(t) x(t)
$$

then $\|x(t)\|$ and $\|y(t)\|$ are bounded for all $t>0$, and $x(t)$ converges to zero asymptotically. 


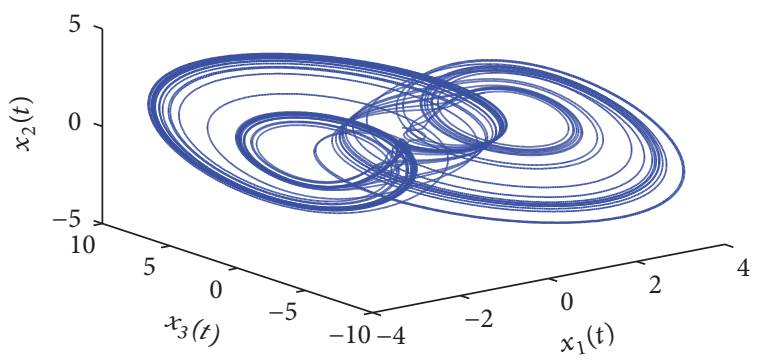

FIgURE 2: Fractional-order Arneodo system.

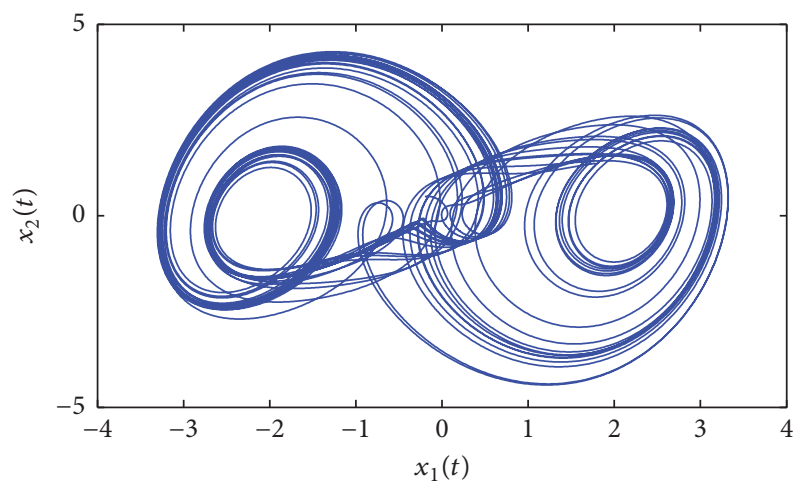

FIGURE 3: The chaotic behavior of fractional-order Arneodo system in $x_{1}-x_{2}$ plane.

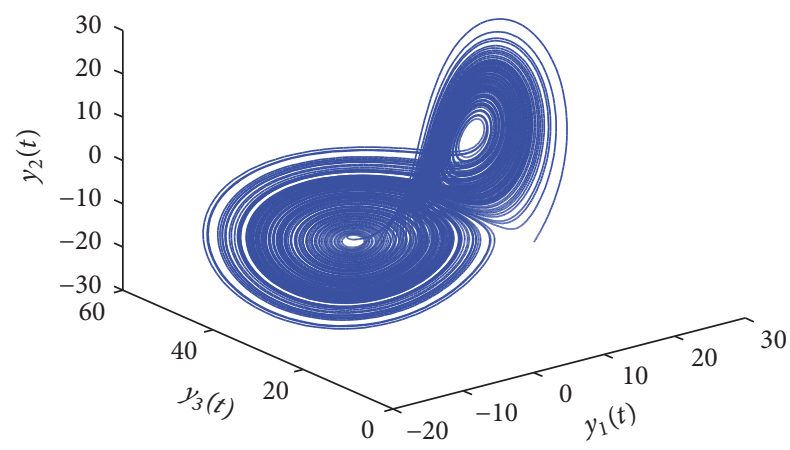

FIGURE 4: Fractional-order Lorenz system.

Proof. From (36), we have

$$
D_{t}^{\alpha} V_{2}(t) \leqslant-h x^{T}(t) x(t) \leqslant 0 .
$$

Based on Lemma 11, we know that $V_{2}(t)$ is monotonically decreasing in $[0,+\infty]$. Then

$$
V_{2}(t) \leqslant V_{2}(0) .
$$

Thus, $\|x(t)\| \leqslant \sqrt{2 V_{2}(0)}$ and $\|y(t)\| \leqslant \sqrt{2 V_{2}(0)}$; namely, $\|x(t)\|$ and $\|y(t)\|$ are bounded. Next we will prove that $x(t)$ tends to 0 asymptotically. Taking $\alpha$ th integral on both sides of (37), we have

$$
V_{2}(t)-V_{2}(0) \leqslant-h D_{t}^{-\alpha} x^{T}(t) x(t) .
$$

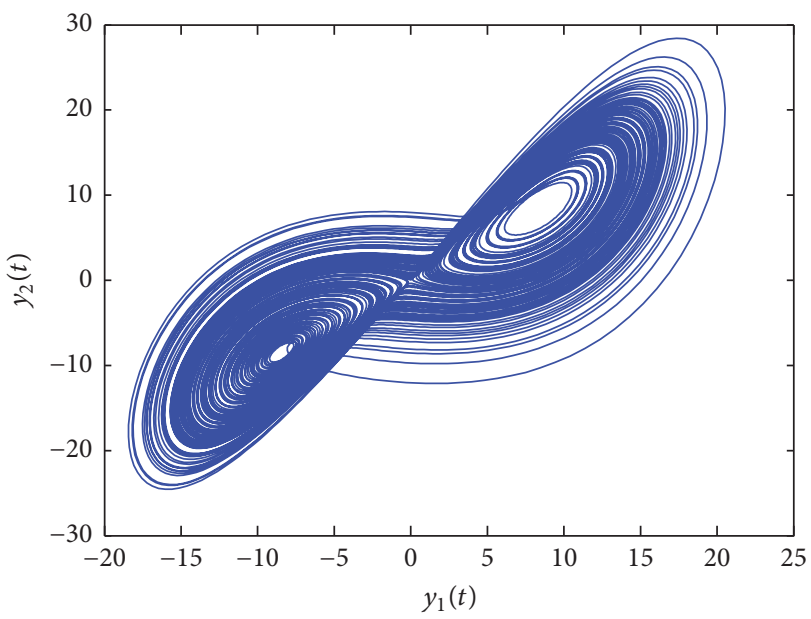

FIGURE 5: The chaotic behavior of fractional-order Lorenz system in $y_{1}-y_{2}$ plane.

Noting that $V_{2}(t)=(1 / 2) x^{T}(t) x(t)+(1 / 2) y^{T}(t) y(t)$, we have $x^{T}(t) x(t) \leqslant 2 V_{2}(t)$. Consequently,

$$
x^{T}(t) x(t) \leqslant 2 V_{2}(0)-2 h D_{t}^{-\alpha} x^{T}(t) x(t) .
$$

Thus, we can find a nonnegative function $Z(t)$ such that

$$
x^{T}(t) x(t)+Z(t)=2 V_{2}(0)-2 h D_{t}^{-\alpha} x^{T}(t) x(t) .
$$

Taking Laplace transform on both sides of (41), we have

$$
X^{T}(s) X(s)=2 V_{2}(0) \frac{s^{\alpha-1}}{s^{\alpha}+2 h}-\frac{s^{\alpha}}{s^{\alpha}+2 h} Z(s) .
$$

According to (30), the solution of (42) is

$$
\begin{aligned}
x^{T}(t) x(t)= & 2 V_{2}(0) E_{\alpha, 0}\left(-2 h t^{\alpha}\right)-Z(t) \\
& *\left[t^{-1} E_{\alpha, 0}\left(-2 h t^{\alpha}\right)\right],
\end{aligned}
$$

where $*$ is convolution. Because $t^{-1}$ and $E_{\alpha, 0}\left(-2 h t^{\alpha}\right)$ are nonnegative functions, it follows from Lemma 12 that $x(t)$ converges to zero asymptotically. This completes the proof of Lemma 13.

Remark 14. If $x^{T}(t) x(t) \leqslant 2 V_{2}(0) E_{\alpha}\left(-2 h t^{\alpha}\right)$, we know that $x(t)$ will tend to 0 asymptotically according to the results in [48]. Namely, $\lim _{t \rightarrow \infty}\|x(t)\|=0$.

\subsection{Stability Analysis}

Theorem 15. If $d(t)=0$, we can realize the synchronization of system (12) and (13) under the effect of the adaptive controller (24) and the fractional-order adaptive law (25). And all the variables in the closed-loop system remain bounded. 


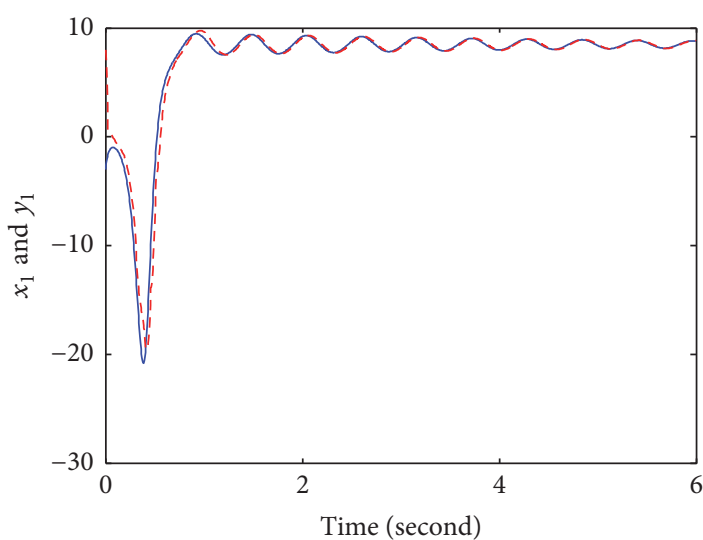

(a)

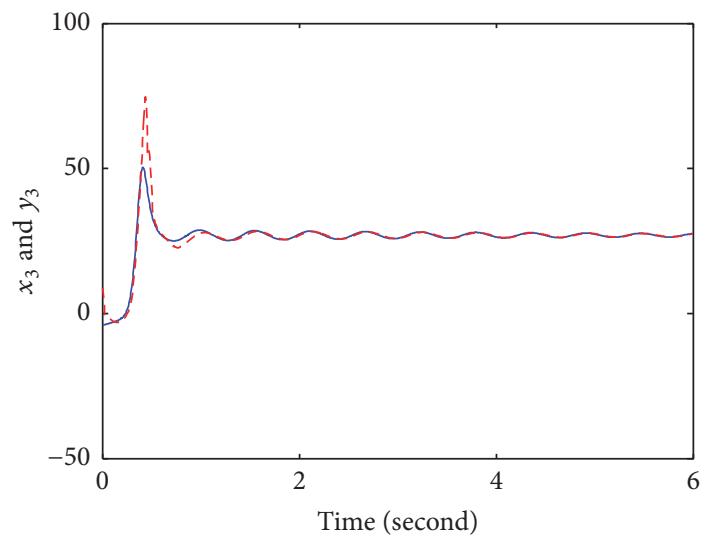

(c)

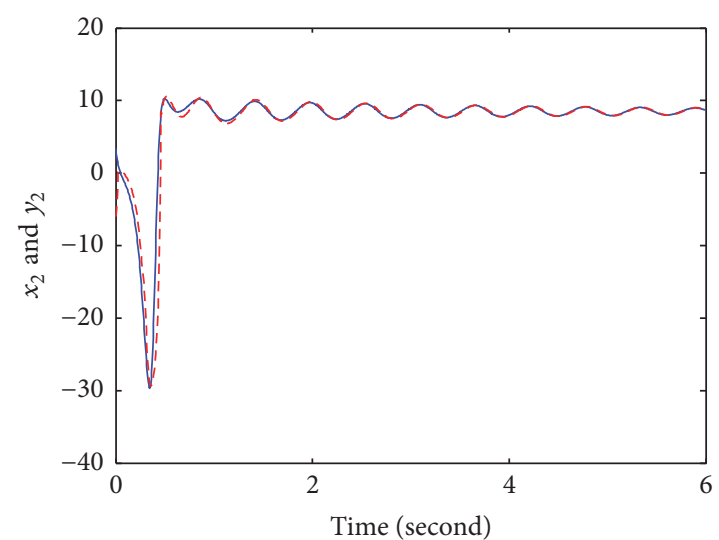

(b)

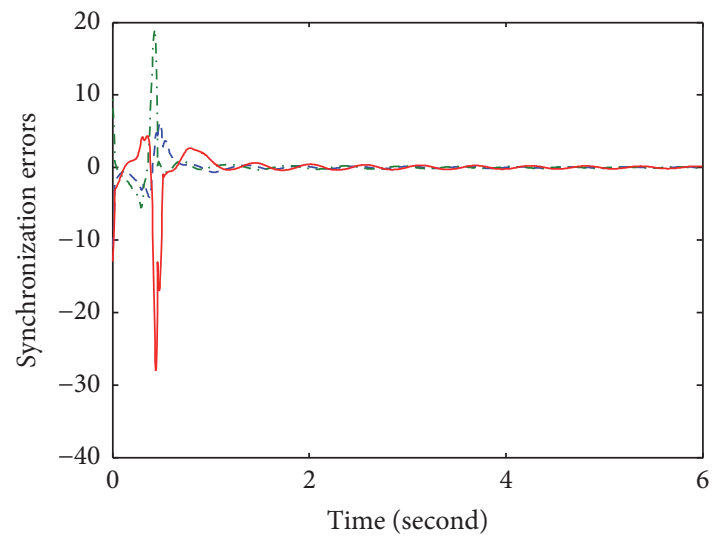

(d)

Figure 6: Synchronization results: (a) $x_{1}(t)$ (solid line) and $y_{1}(t)$ (dashed-dotted line); (b) $x_{2}(t)$ (solid line) and $y_{2}(t)$ (dashed-dotted line); and $(\mathrm{c}) x_{3}(t)$ (solid line) and $y_{3}(t)$ (dashed-dotted line). (d) Synchronization errors: $e_{1}(t)$ (dashed line), $e_{2}(t)$ (dashed-dotted line), and $e_{3}(t)$ (solid line).

Proof. Substituting the synchronization controllers (24) and (23) into the error dynamical equation (19), we have

$$
\begin{aligned}
D_{t}^{\alpha} e(t)= & C e(t)+h(t)-K e(t)-\mathscr{W}^{T} \varphi(x) \\
& -H \operatorname{sign}(e(t)) \\
= & -(K-C) e(t)+\epsilon(t)-\widetilde{\mathscr{W}}^{T} \varphi(x) \\
& -H \operatorname{sign}(e(t)) \\
= & -P e(t)+\epsilon(t)-\widetilde{\mathscr{W}}^{T} \varphi(x)-H \operatorname{sign}(e(t)),
\end{aligned}
$$

where $P=K-C$. We can choose an appropriate gain matrix $K$ such that $P$ is a positive definite matrix. Multiplying $e^{T}(t)$ on both sides of (44) yields

$$
\begin{aligned}
e^{T}(t) D_{t}^{\alpha} e(t)= & -e^{T}(t) P e(t)+e^{T}(t) \epsilon(t) \\
& -e^{T}(t) \widetilde{\mathscr{W}}^{T} \varphi(x) \\
& -e^{T}(t) H \operatorname{sign}(e(t))
\end{aligned}
$$

$$
\begin{aligned}
\leqslant & -e^{T}(t) P e(t)+\sum_{i=1}^{n}\left|e_{i}(t)\right| \epsilon_{i}^{*}(t) \\
& -\sum_{i=1}^{n} e_{i}(t) \widetilde{\mathscr{W}}_{i}^{T} \varphi_{i}(x) \\
& -\sum_{i=1}^{n}\left|e_{i}(t)\right| \epsilon_{i}^{*}(t) \\
\leqslant & -e^{T}(t) P e(t)-\sum_{i=1}^{n} e_{i}(t) \widetilde{\mathscr{W}}_{i}^{T} \varphi_{i}(x) .
\end{aligned}
$$

Consider the following Lyapunov function:

$$
V(t)=\frac{1}{2} e^{T}(t) e(t)+\frac{1}{2} \sum_{i=1}^{n} \frac{1}{\mu_{i}} \widetilde{\mathscr{W}}_{i}^{T} \widetilde{\mathscr{W}}_{i} .
$$

Because the $\alpha$ th Caputo derivative of a constant is 0 , we have $D_{t}^{\alpha} \widetilde{W}_{i}=D_{t}^{\alpha} W_{i}$. Taking $\alpha$ th derivative of $V(t)$ based on Lemma 10 gives

$$
D_{t}^{\alpha} V(t) \leqslant e^{T}(t) D_{t}^{\alpha} e(t)+\sum_{i=1}^{n} \frac{1}{\mu_{i}} \widetilde{\mathscr{W}}_{i}^{T} D_{t}^{\alpha} \mathscr{W}_{i} .
$$




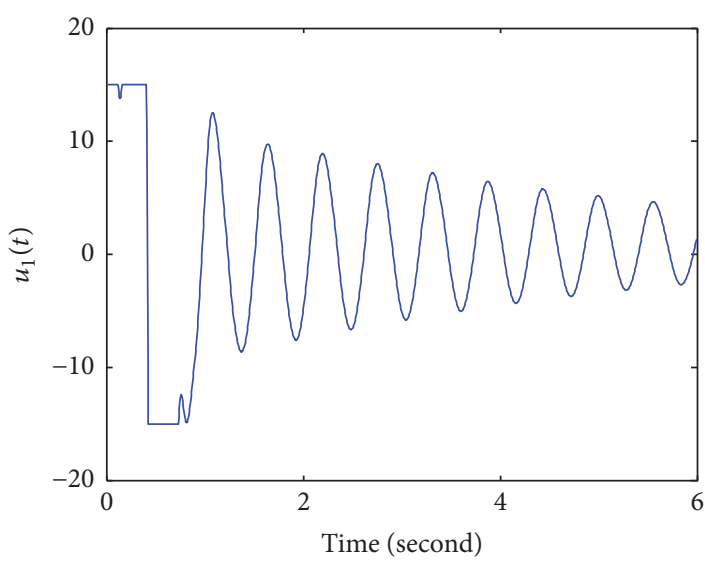

(a)

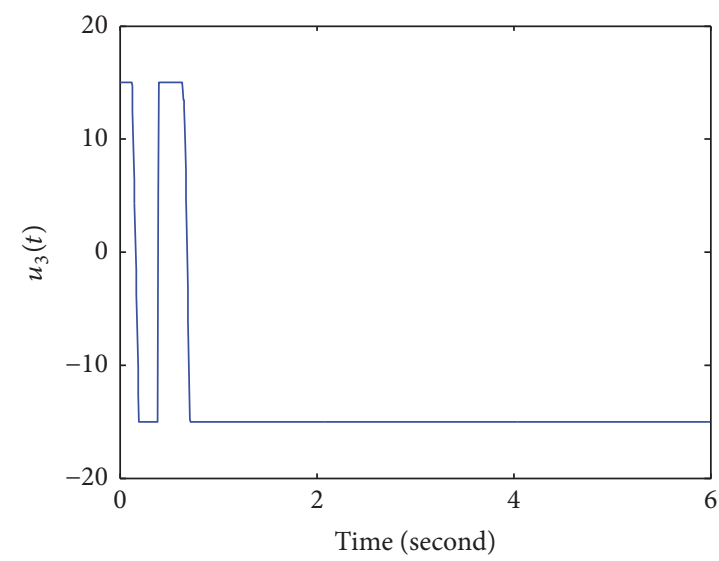

(c)

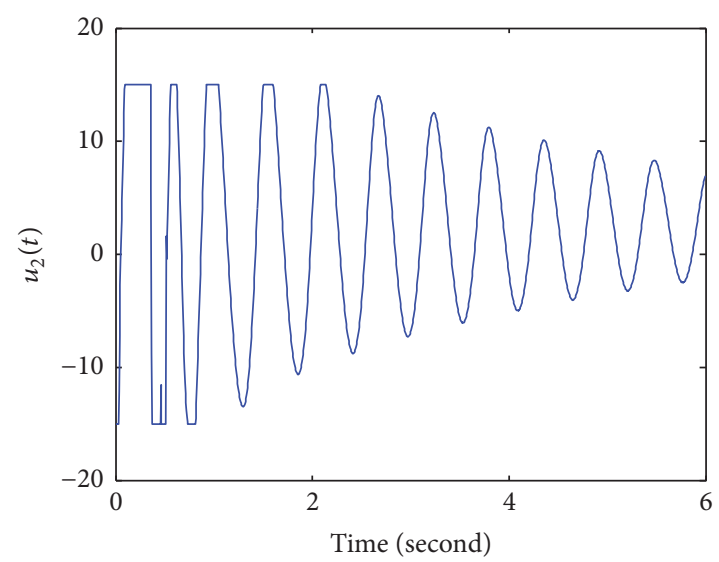

(b)

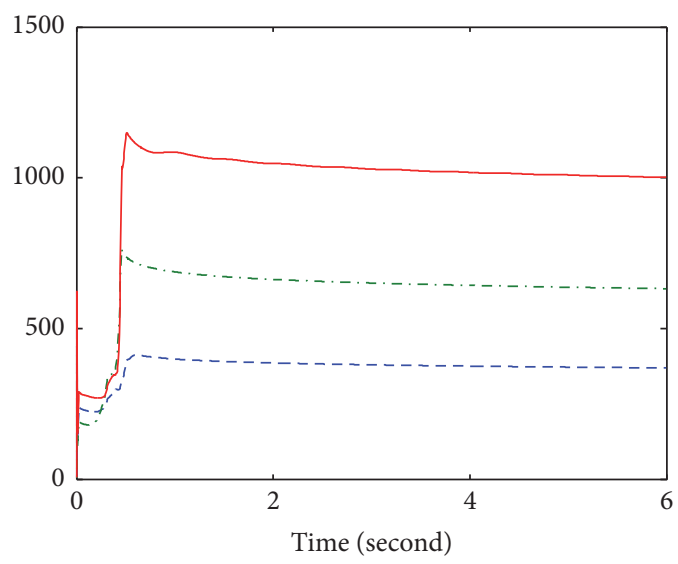

(d)

Figure 7: Control inputs and parameters: (a) $u_{1}(t)$; (b) $u_{2}(t)$; (c) $u_{3}(t)$; and (d) $\left\|W_{1}(t)\right\|$ (dashed line), $\left\|W_{2}(t)\right\|$ (dashed-dotted line), and $\left\|W_{3}(t)\right\|$ (solid line).

Substituting (45) and (25) into (47), we have

$$
\begin{aligned}
D_{t}^{\alpha} V(t) \leqslant & -e^{T}(t) P e(t)-\sum_{i=1}^{n} e_{i}(t) \widetilde{\mathscr{W}}_{i}^{T} \varphi_{i}(x) \\
& +\sum_{i=1}^{n} \frac{1}{\mu_{i}} \widetilde{\mathscr{W}}_{i}^{T} D_{t}^{\alpha} \mathscr{W}_{i} \\
\leqslant & -e^{T}(t) P e(t)-\sum_{i=1}^{n} e_{i}(t) \widetilde{\mathscr{W}}_{i}^{T} \varphi_{i}(x) \\
& +\sum_{i=1}^{n} e_{i}(t) \widetilde{\mathscr{W}}_{i}^{T} \varphi_{i}(x) \leqslant-e^{T}(t) P e(t) \\
\leqslant & -\lambda_{\min } e^{T}(t) e(t),
\end{aligned}
$$

where $\lambda_{\min }$ is the minimum eigenvalue of matrix $P$. From (48) and Lemma 13, we know that the synchronization error $e(t)$ tends to 0 asymptotically; namely, $\lim _{t \rightarrow \infty}\|e(t)\|=0$.

Theorem 16. We can realize the synchronization of system (12) and (13) under the effect of the adaptive controller (24) and the fractional-order adaptive laws (see (25) and (26)). And all variables in the closed-loop system remain bounded when $d(t) \neq 0$.

Proof. Substituting (23) and (24) into the error dynamical equation (19), we obtain

$$
\begin{aligned}
D_{t}^{\alpha} e(t)= & C e(t)+h(t)+d(t)-K e(t)-\mathscr{W}^{T} \varphi(x) \\
& -H \operatorname{sign}(e(t))-\hat{\rho} \operatorname{sign}(e(t)) \\
= & -(K-C) e(t)+d(t)+\epsilon(t)-\widetilde{\mathscr{W}}^{T} \varphi(x) \\
& -H \operatorname{sign}(e(t))-\hat{\rho} \operatorname{sign}(e(t)) \\
\leqslant & -P e(t)+\epsilon(t)-\widetilde{\mathscr{W}}^{T} \varphi(x)-H \operatorname{sign}(e(t)) \\
& -\tilde{\rho} \operatorname{sign}(e(t)),
\end{aligned}
$$

where $P=K-C$ is a positive matrix.

$$
\tilde{\rho}_{i}(t)=\widehat{\rho}_{i}(t)-\rho_{i} .
$$




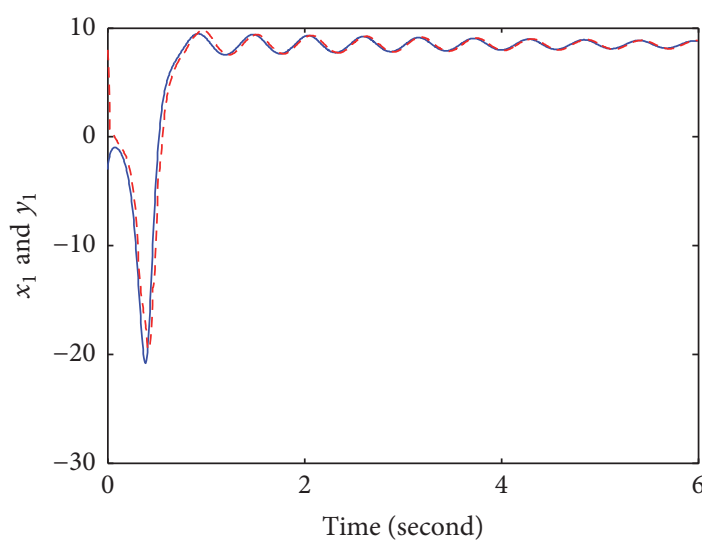

(a)

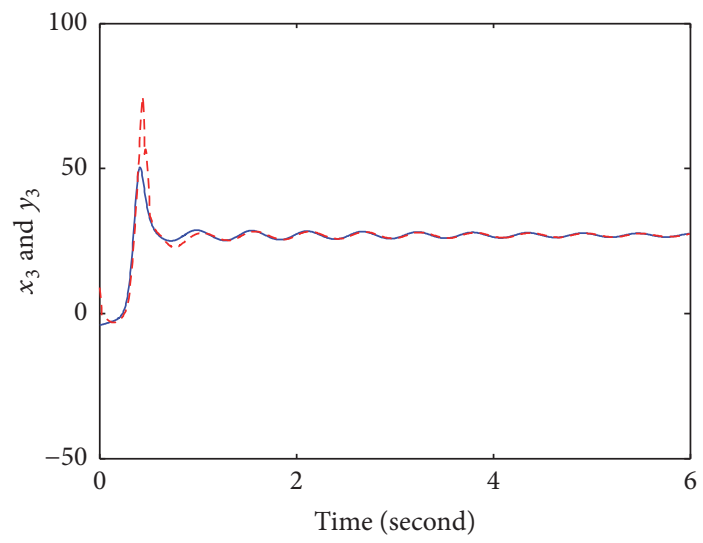

(c)

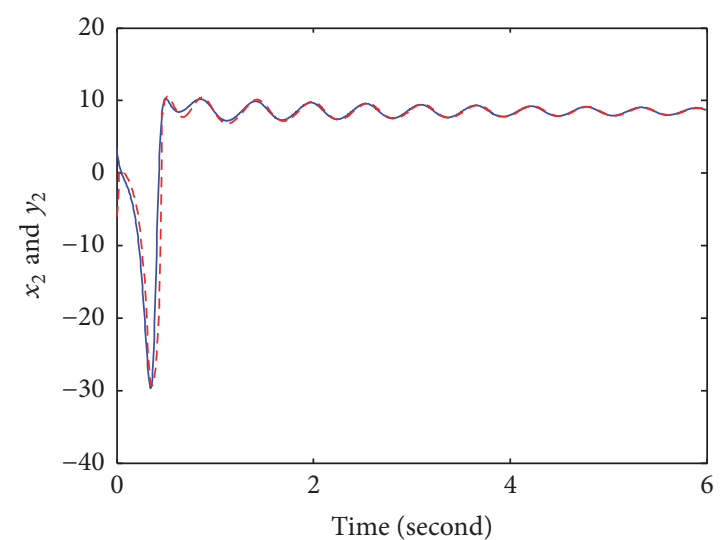

(b)

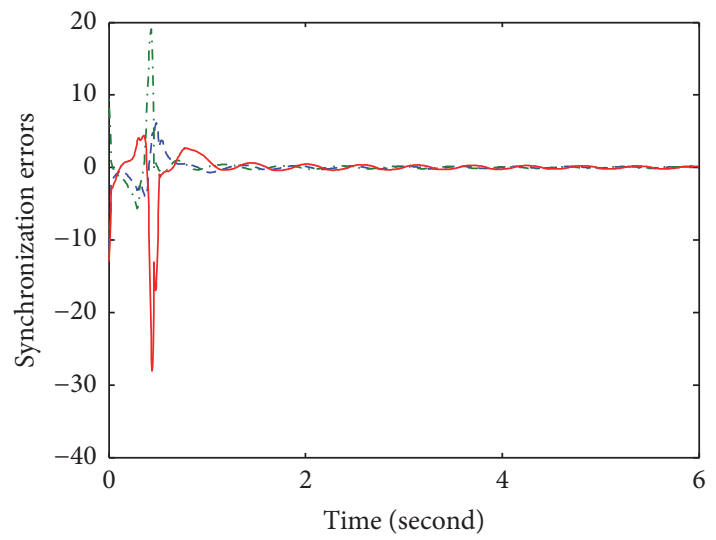

(d)

FIGURE 8: Synchronization results: (a) $x_{1}(t)$ (solid line) and $y_{1}(t)$ (dashed-dotted line); (b) $x_{2}(t)$ (solid line) and $y_{2}(t)$ (dashed-dotted line); and $(\mathrm{c}) x_{3}(t)$ (solid line) and $y_{3}(t)$ (dashed-dotted line). (d) Synchronization errors: $e_{1}(t)$ (dashed line), $e_{2}(t)$ (dashed-dotted line), and $e_{3}(t)$ (solid line).

Multiplying $e^{T}(t)$ on both sides of (50) gives

$$
\begin{aligned}
e^{T}(t) D_{t}^{\alpha} e(t)= & -e^{T}(t) \operatorname{Pe}(t)+e^{T}(t) \epsilon(t) \\
& -e^{T}(t) \widetilde{\mathscr{W}}^{T} \varphi(x) \\
& -e^{T}(t) H \operatorname{sign}(e(t)) \\
& -e^{T}(t) \widetilde{\rho} \operatorname{sign}(e(t)) \\
\leqslant & -e^{T}(t) P e(t)+\sum_{i=1}^{n}\left|e_{i}(t)\right| \epsilon_{i}^{*}(t) \\
& -\sum_{i=1}^{n} e_{i}(t) \widetilde{\mathscr{W}}_{i}^{T} \varphi_{i}(x)-\sum_{i=1}^{n} \epsilon_{i}^{*}(t)\left|e_{i}(t)\right| \\
& -\sum_{i=1}^{n} \widetilde{\rho}_{i}(t)\left|e_{i}(t)\right| \\
\leqslant & -e^{T}(t) P e(t)-\sum_{i=1}^{n} e_{i}(t) \widetilde{\mathscr{W}}_{i}^{T} \varphi_{i}(x) \\
& -\sum_{i=1}^{n} \widetilde{\rho}_{i}(t)\left|e_{i}(t)\right| .
\end{aligned}
$$

Consider the following Lyapunov function:

$$
V(t)=\frac{1}{2} e^{T}(t) e(t)+\frac{1}{2} \sum_{i=1}^{n} \frac{1}{\mu_{i}} \widetilde{\mathscr{W}}_{i}^{T} \widetilde{\mathscr{W}}_{i}+\frac{1}{2} \sum_{i=1}^{n} \frac{1}{\gamma_{i}} \widetilde{\rho}_{i}^{2}(t) .
$$

Taking $\alpha$ th derivative of $V(t)$ based on Lemma 10, we have

$$
\begin{aligned}
D_{t}^{\alpha} V(t) \leqslant & e^{T}(t) D_{t}^{\alpha} e(t)+\sum_{i=1}^{n} \frac{1}{\mu_{i}} \widetilde{\mathscr{W}}_{i}^{T} D_{t}^{\alpha} \mathscr{W}_{i} \\
& +\sum_{i=1}^{n} \frac{1}{\gamma_{i}} \widetilde{\rho}_{i}(t) D_{t}^{\alpha} \rho_{i}(t) .
\end{aligned}
$$

Substituting (45), (25), and (26) into (53), we have

$$
\begin{aligned}
D_{t}^{\alpha} V(t) \leqslant & -e^{T}(t) P e(t)-\sum_{i=1}^{n} e_{i}(t) \widetilde{\mathscr{W}}_{i}^{T} \varphi_{i}(x) \\
& -\sum_{i=1}^{n} \widetilde{\rho}_{i}(t)\left|e_{i}(t)\right|+\sum_{i=1}^{n} \frac{1}{\mu_{i}} \widetilde{\mathscr{W}}_{i}^{T} D_{t}^{\alpha} \mathscr{W}_{i} \\
& +\sum_{i=1}^{n} \frac{1}{\gamma_{i}} \widetilde{\rho}_{i}(t) D_{t}^{\alpha} \rho_{i}(t)
\end{aligned}
$$






(a)



(c)



(b)

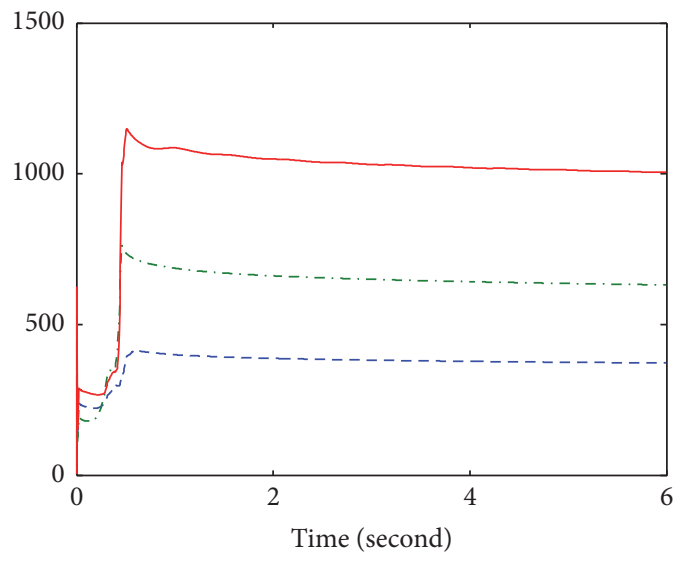

(d)

FIGURE 9: Control inputs and parameters: (a) $u_{1}(t)$; (b) $u_{2}(t)$; (c) $u_{3}(t)$; and (d) $\left\|W_{1}(t)\right\|$ (dashed line), $\left\|W_{2}(t)\right\|$ (dotted dashed line), and $\left\|W_{3}(t)\right\|$ (solid line).

$$
\begin{aligned}
\leqslant & -e^{T}(t) P e(t)-\sum_{i=1}^{n} e_{i}(t) \widetilde{\mathscr{W}}_{i}^{T} \varphi_{i}(x) \\
& -\sum_{i=1}^{n} \widetilde{\rho}_{i}(t)\left|e_{i}(t)\right|+\sum_{i=1}^{n} e_{i}(t) \widetilde{\mathscr{W}}_{i}^{T} \varphi_{i}(x) \\
& +\sum_{i=1}^{n} \widetilde{\rho}_{i}(t)\left|e_{i}(t)\right| \leqslant-e^{T}(t) P e(t) \\
\leqslant & -\lambda_{\min } e^{T}(t) e(t)
\end{aligned}
$$

where $\lambda_{\text {min }}$ is the minimum eigenvalue of matrix $P$. From (54) and Lemma 13, we know that the synchronization error $e(t)$ tends to 0 asymptotically; namely, $\lim _{t \rightarrow \infty}\|e(t)\|=0$.

\section{Simulation Studies}

In this section, two fractional-order chaotic systems and two fractional-order hyperchaotic systems will be synchronized to show the validity of the above method.
4.1. Example 1. Choose the frequently used fractional-order Arneodo system [49] in literature as the drive system:

$$
\begin{aligned}
& D_{t}^{\alpha} x_{1}(t)=x_{2}(t) \\
& D_{t}^{\alpha} x_{2}(t)=x_{3}(t) \\
& D_{t}^{\alpha} x_{3}(t)=a x_{1}(t)-b x_{2}(t)-c x_{3}(t)+d x_{1}^{3}(t) .
\end{aligned}
$$

When $a=-5.5, b=3.5, c=0.4, d=-1$, and $\alpha=0.9$, the fractional-order Arneodo system shows chaotic phenomenon, which is indicated in Figures 2 and 3.

Let the response system be the following fractional-order Lorenz system [50]:

$$
\begin{aligned}
& D_{t}^{\alpha} y_{1}(t)=\sigma\left(y_{2}(t)-y_{1}(t)\right) \\
& D_{t}^{\alpha} y_{2}(t)=y_{1}(t)\left(\rho-y_{3}(t)\right)-y_{2}(t) \\
& D_{t}^{\alpha} y_{3}(t)=y_{1}(t) y_{2}(t)-\beta y_{3}(t) .
\end{aligned}
$$

When $\sigma=10, \rho=28, \beta=8 / 3$, and $\alpha=0.99$, the chaotic behavior of system (56) is included in Figures 4 and 5. 


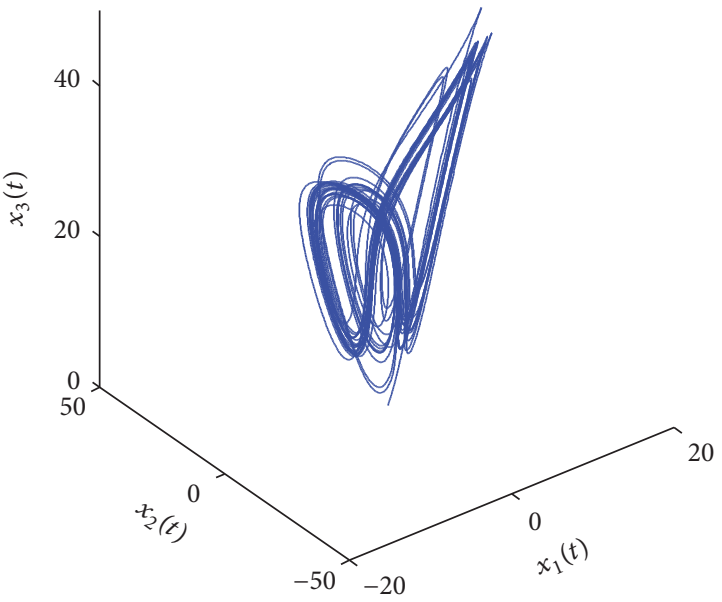

(a)

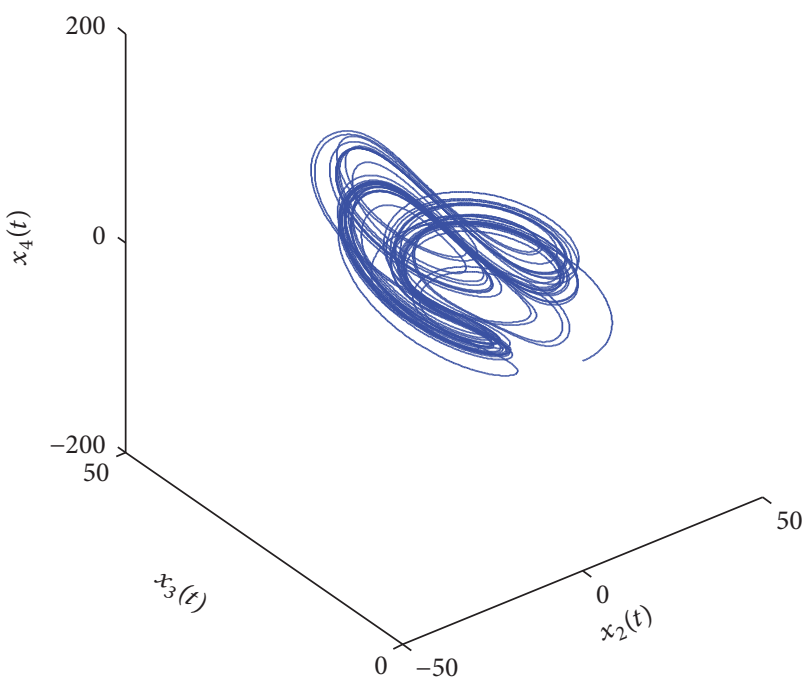

(c)



(b)

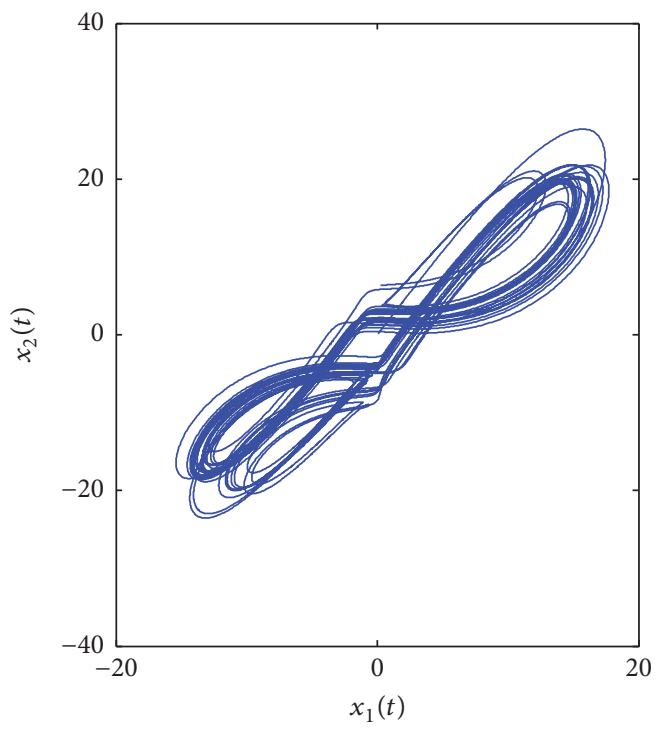

(d)

FIGURE 10: Fractional-order hyperchaotic Lorenz system (58).

It follows from (55) and (56) that

$$
\begin{aligned}
& A=\left(\begin{array}{ccc}
0 & 0 & 0 \\
0 & 0 & 1 \\
a & -b & -c
\end{array}\right) \\
& C=\left(\begin{array}{ccc}
-\sigma & \sigma & 0 \\
\rho & -1 & 0 \\
0 & 0 & -\beta
\end{array}\right) .
\end{aligned}
$$

Firstly, we consider the synchronization of system (55) and system (56) in the absence of external disturbance (namely, $d(t)=0$ ). In the simulation, we choose $\sigma=0.8$, $\alpha=0.96$, and $k_{1}=k_{2}=k_{3}=3$. The initial condition of system (55) is $x(0)=[-3,3.4,-4]^{T}$, and the initial condition of system $(56)$ is $y(0)=[8,-6,9]^{T}$. With respect to the fuzzy logic system, we define 6 Gaussian membership functions uniformly distributed on [-20 20]. The initial condition of the fuzzy parameter is chosen as zero vector. The input saturation limiter $u_{r}=-u_{l}=5$. The simulation results are depicted in Figures 6 and 7.

Then we study the synchronization of system (55) and system (56) with the external disturbance. In simulation, we select $\sigma=0.8, \alpha=0.96$, and $k_{1}=k_{2}=k_{3}=3$. The initial conditions are $x(0)=[-3,3.4,-4]^{T}$ and $y(0)=[8,-6,9]^{T}$. Let $d(t)=[0.15 \sin (t), 0.05 \cos (t), 0.1 \cos (t)]^{T}$ and let the input saturation limiter be $u_{r}=-u_{l}=5$. The simulation results are as shown in Figures 8 and 9.

4.2. Example 2. Choose the fractional-order hyperchaotic Lorenz system [50] as the drive system: 


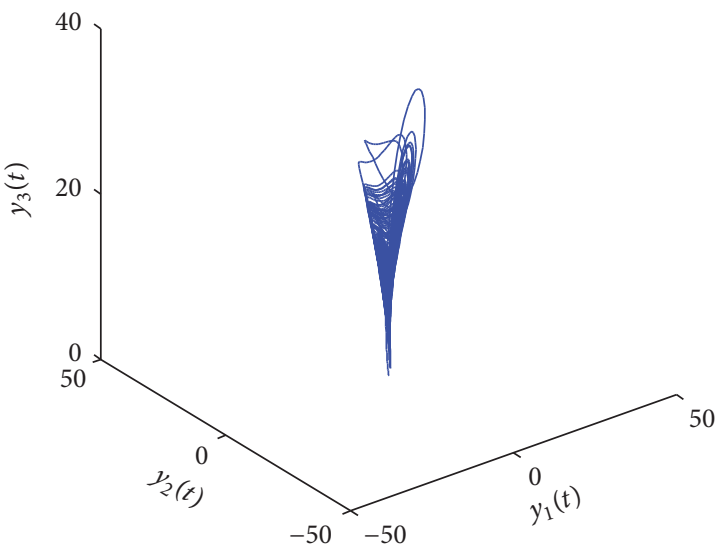

(a)



(c)



(b)



(d)

FIGURE 11: Fractional-order hyperchaotic system (59).

$$
\begin{aligned}
& D_{t}^{\alpha} x_{1}(t)=a\left(x_{2}(t)-x_{1}(t)\right)+x_{4}(t) \\
& D_{t}^{\alpha} x_{2}(t)=c x_{1}(t)-x_{2}(t)-x_{1}(t) x_{3}(t) \\
& D_{t}^{\alpha} x_{3}(t)=x_{1}(t) x_{2}(t)-b x_{3}(t) \\
& D_{t}^{\alpha} x_{4}(t)=-x_{1}(t) x_{3}(t)+\gamma x_{4}(t) .
\end{aligned}
$$

When $a=10, b=8 / 3, c=28, \gamma=-1$, and $\alpha=0.93$, the fractional-order hyperchaotic Lorenz system (58) has chaotic attractors, as shown in Figure 10.

The response system is the following fractional-order hyperchaotic system:

$$
\begin{aligned}
& D_{t}^{\alpha} y_{1}(t)=a\left(y_{2}(t)-y_{1}(t)\right) \\
& D_{t}^{\alpha} y_{2}(t)=d y_{1}(t)-y_{1}(t) y_{3}(t)+c y_{2}(t)-y_{4}(t) \\
& D_{t}^{\alpha} y_{3}(t)=y_{1}(t) y_{2}(t)-b y_{3}(t) \\
& D_{t}^{\alpha} y_{4}(t)=y_{1}(t)+k .
\end{aligned}
$$

When $a=36.01, b=3.00, c=28.00, d=-16.00, k=0.50$, and $\alpha=0.95$, the chaotic behavior of system (59) is given in Figure 11.
Firstly, we consider the synchronization of system (58) and system (59) when $d(t)=0$. In the simulation, we choose the control parameters as $\sigma=0.8, \alpha=0.95$, and $k_{1}=k_{2}=k_{3}=k_{4}=3$. The initial conditions are $x(0)=[-6,2.4,-4,-6]^{T}$ and $y(0)=[9.6,-6,9,5]^{T}$. The input saturation limiter $u_{r 1}=-u_{l 1}=12$ and $u_{r i}=-u_{l i}=14$, $i=2,3,4$. The simulation results are presented in Figures $12-15$.

Then, we consider the synchronization of system (58) and system (59) with external disturbance. In the simulation, let $\sigma=0.8, \alpha=0.95, k_{1}=k_{2}=k_{3}=k_{4}=3$, and $d(t)=[0.15 \sin (t), 0.05 \cos (t), 0.1 \cos (t), 0.5 \sin (t) \cos (t)]^{T}$. The initial conditions are $x(0)=[-3,3.4,-4,-6]^{T}$ and $y(0)=$ $[9.6,-6,9,5]^{T}$. The input saturation limiter is $u_{r 1}=-u_{l 1}=12$ and $u_{r i}=-u_{l i}=14, i=2,3,4$. The simulation results are depicted in Figures 16-19.

\section{Conclusions}

In this paper, we investigate the synchronization for two uncertain fractional-order nonlinear chaotic systems with saturated input and external disturbances in accordance with 




(a)



(c)

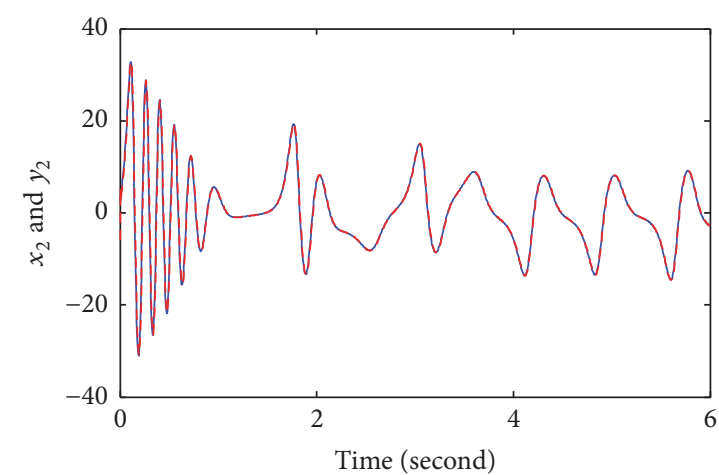

(b)



(d)

Figure 12: Synchronization results: (a) $x_{1}(t)$ (solid line) and $y_{1}(t)$ (dashed line); (b) $x_{2}(t)$ (solid line) and $y_{2}(t)$ (dashed line); (c) $x_{3}(t)$ (solid line) and $y_{3}(t)$ (dashed line); and (d) $x_{4}(t)$ (solid line) and $y_{4}(t)$ (dashed line).

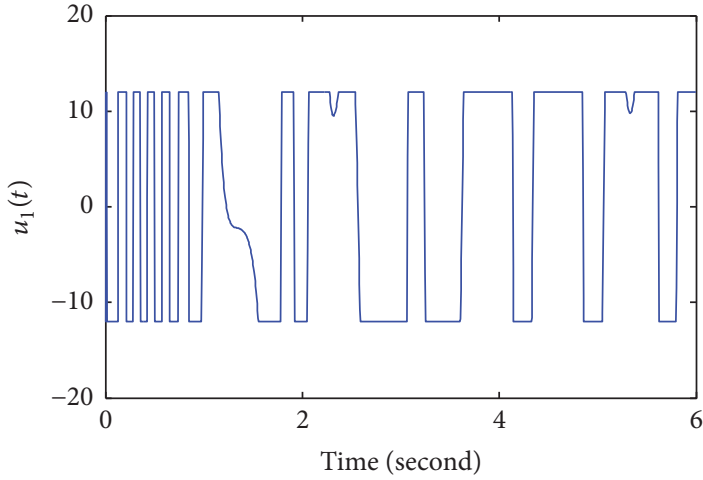

(a)

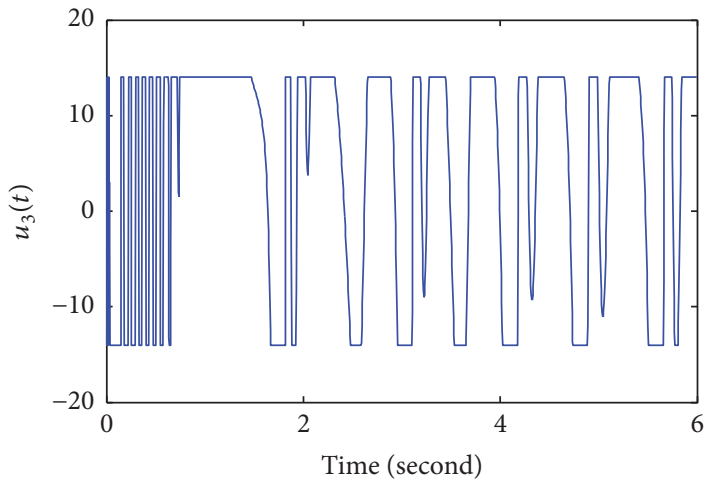

(c)

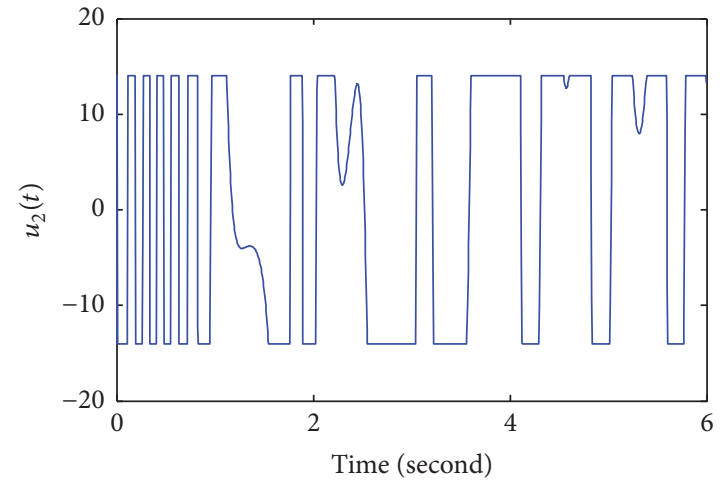

(b)

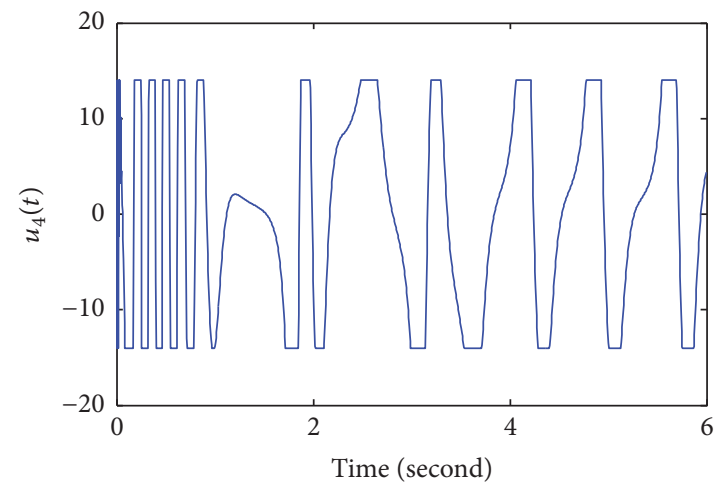

(d)

FIGURE 13: Control inputs: (a) $u_{1}(t)$; (b) $u_{2}(t)$; (c) $u_{3}(t)$; and (d) $u_{4}(t)$. 


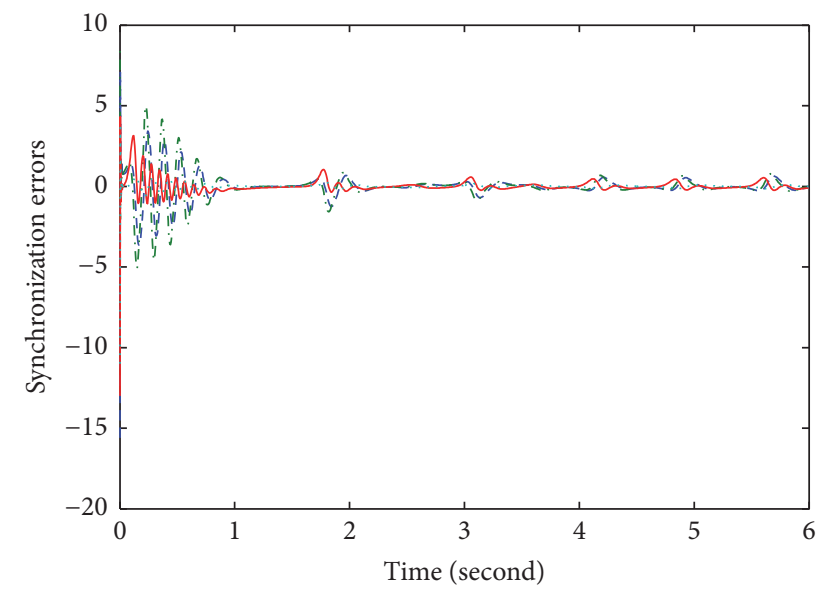

FIGURE 14: Synchronization errors: $e_{1}(t)$ (dotted line), $e_{2}(t)$ (dotted dashed line), $e_{3}(t)$ (solid line), and $e_{4}(t)$ (dashed line).

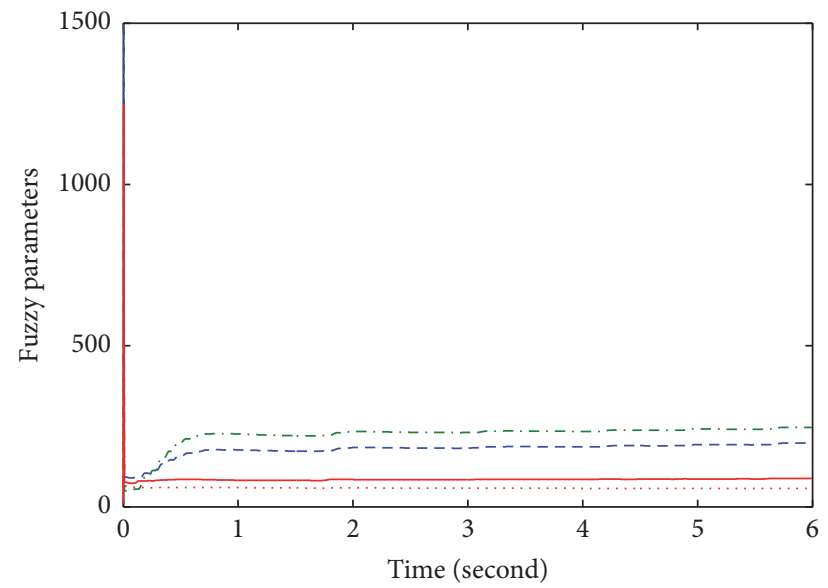

Figure 15: Parameters: $\left\|W_{1}(t)\right\|$ (dotted line), $\left\|W_{2}(t)\right\|$ (dotted dashed line), $\left\|W_{3}(t)\right\|$ (solid line), and $\left|W_{4}(t)\right|$ (dashed line).

the fractional Lyapunov stability theory and adaptive fuzzy control method. A fractional-order adaptive controller that can guarantee that the synchronization error tends to a small region of origin and fractional parameters adaptive laws are designed. Based on the proposed method, we can achieve synchronization of many fractional-order chaotic systems and hyperchaotic systems. It should be mentioned that the proposed controller can guarantee that the synchronization errors converge to a small region of the origin eventually. How to design an adaptive fuzzy controller such that the dynamical system is asymptotic stable is one of our future research directions.

\section{Conflicts of Interest}

The authors declare that there are no conflicts of interest regarding the publication of this paper.

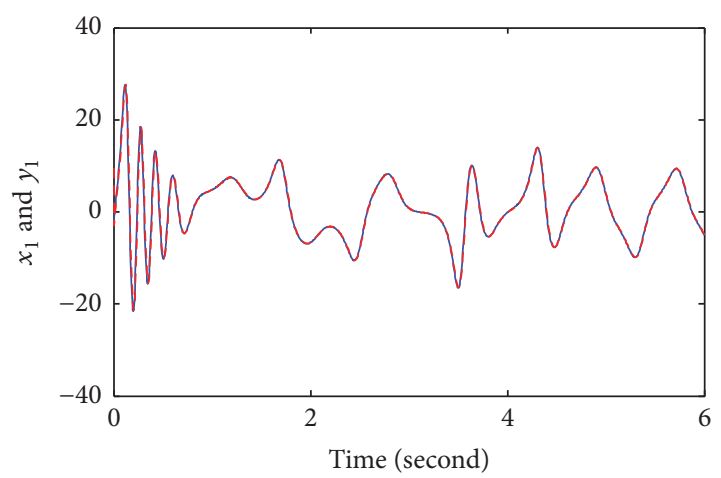

(a)



(b)

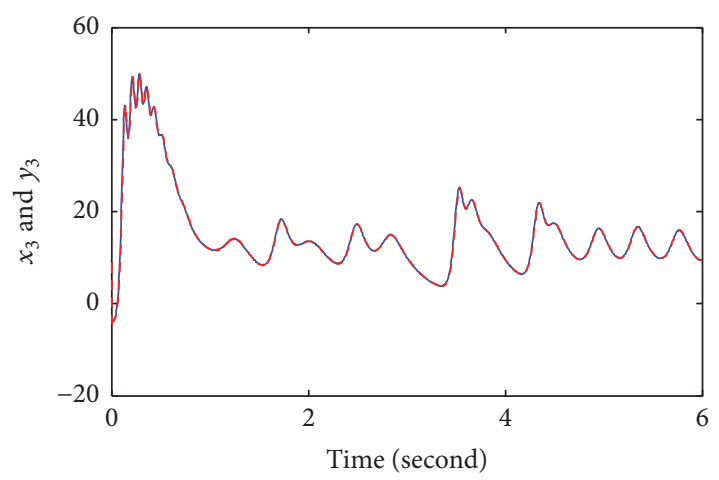

(c)



(d)

FIGURE 16: Synchronization results: (a) $x_{1}(t)$ (solid line) and $y_{1}(t)$ (dashed line); (b) $x_{2}(t)$ (solid line) and $y_{2}(t)$ (dashed line); (c) $x_{3}(t)$ (solid line) and $y_{3}(t)$ (dashed line); and (d) $x_{4}(t)$ (solid line) and $y_{4}(t)$ (dashed line). 


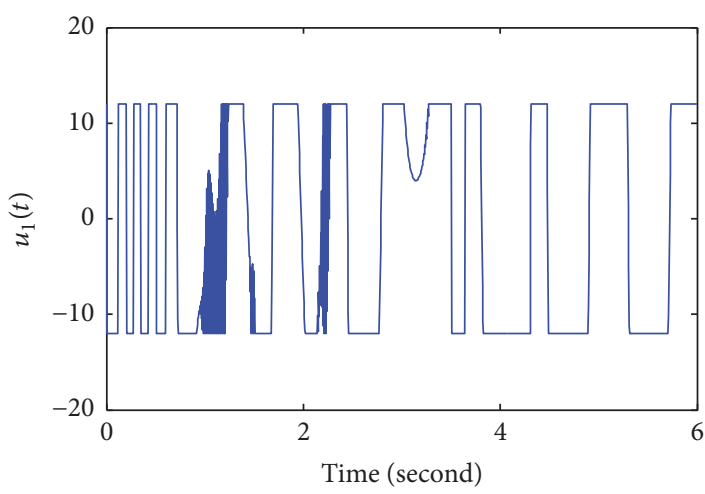

(a)



(c)



(b)

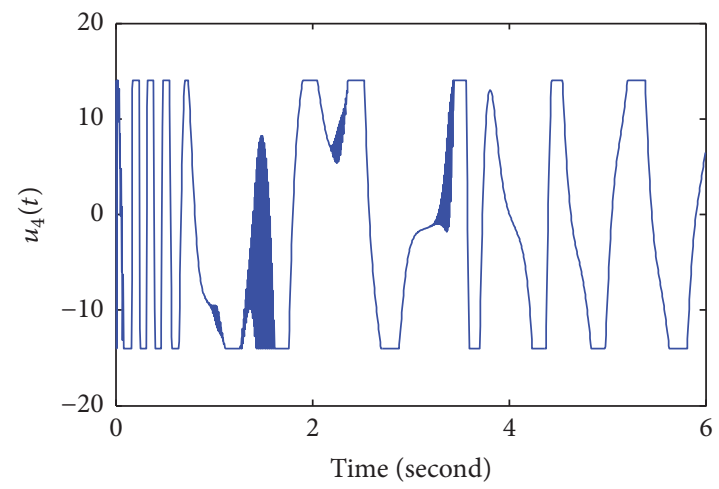

(d)

FIGURE 17: Control inputs: (a) $u_{1}(t)$; (b) $u_{2}(t)$; (c) $u_{3}(t)$; and (d) $u_{4}(t)$.

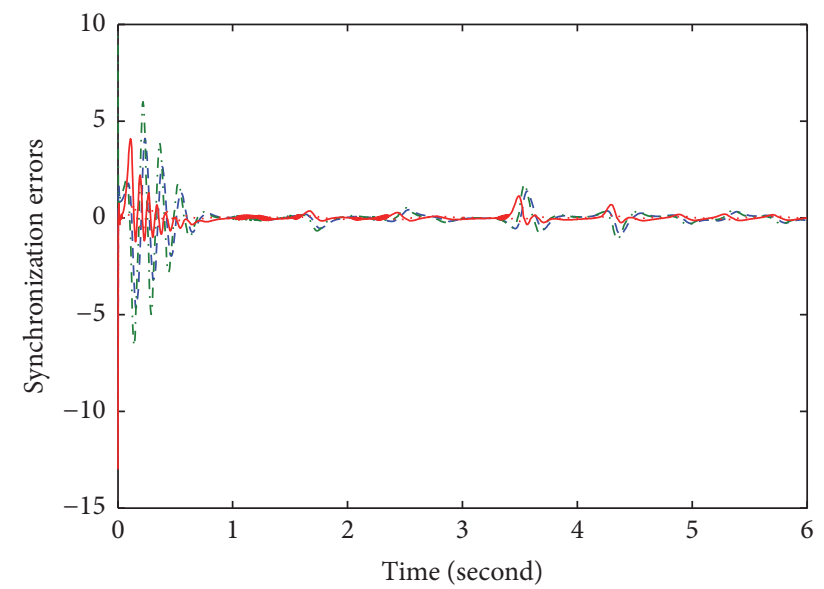

FIGURE 18: Synchronization errors: $e_{1}(t)$ (dotted line), $e_{2}(t)$ (dot dash line), $e_{3}(t)$ (solid line), and $e_{4}(t)$ (dashed line).

\section{Acknowledgments}

This work is supported by the National Natural Science Foundation of China (Grants nos. 11771263 and 11601177), the Natural Science Foundation of Anhui Province of China (Grant no. 1508085QA16), and the Foundation for



FIgUre 19: Parameters: $\left\|W_{1}(t)\right\|$ (dotted line), $\left\|W_{2}(t)\right\|$ (dotted dashed line), $\left\|W_{3}(t)\right\|$ (solid line), and $\left\|W_{4}(t)\right\|$ (dashed line).

Distinguished Young Talents in Higher Education of Anhui Province of China (Grant no. GXYQZD2016257).

\section{References}

[1] A. Elsaid, "Homotopy analysis method for solving a class of fractional partial differential equations," Communications in 
Nonlinear Science and Numerical Simulation, vol. 16, no. 9, pp. 3655-3664, 2011.

[2] P. Han and M. E. Schonbek, "Large time decay properties of solutions to a viscous Boussinesq system in a half space," Advances in Differential Equations, vol. 19, no. 1-2, pp. 87-132, 2014.

[3] R. M. Balan and C. A. Tudor, "Stochastic heat equation with multiplicative fractional-colored noise," Journal of Theoretical Probability, vol. 23, no. 3, pp. 834-870, 2010.

[4] A. Elsaid, "Fractional differential transform method combined with the Adomian polynomials," Applied Mathematics and Computation, vol. 218, no. 12, pp. 6899-6911, 2012.

[5] G. S. Frederico and D. F. Torres, "Necessary optimality conditions for fractional action-like problems with intrinsic and observer times," WSEAS Transactions on Mathematics, vol. 7, no. 1, pp. 6-11, 2008.

[6] A. Hussain, M. Faryad, and Q. A. Naqvi, "Fractional curl operator and fractional Chiro-waveguide," Journal of Electromagnetic Waves and Applications, vol. 21, no. 8, pp. 1119-1129, 2007.

[7] M. Zubair, M. J. Mughal, and Q. A. Naqvi, "An exact solution of the spherical wave equation in D-dimensional fractional space," Journal of Electromagnetic Waves and Applications, vol. 25, no. 10, pp. 1481-1491, 2011.

[8] H. Liu, Y. Pan, S. Li, and Y. Chen, "Adaptive Fuzzy Backstepping Control of Fractional-Order Nonlinear Systems," IEEE Transactions on Systems, Man, and Cybernetics: Systems, vol. 47, no. 8, pp. 2209-2217, 2017.

[9] H. Liu, S. Li, J. D. Cao, A. G. Alsaedi, and F. E. Alsaadi, "Adaptive fuzzy prescribed performance controller design for a class of uncertain fractional-order nonlinear systems with external disturbances," Neurocomputing, vol. 219, pp. 422-430, 2017.

[10] H. Liu, S. Li, G. Li, and H. Wang, "Robust adaptive control for fractional-order financial chaotic systems with system uncertainties and external disturbances," Information Technology and Control, vol. 46, no. 2, 2017.

[11] I. Podlubny, Fractional Differential Equations, vol. 198 of Mathematics in Science and Engineering, Academic Press, San Diego, Calif, USA, 1998.

[12] R. Hilfer, Applications of Fractional Calculus in Physics, World Scientific, Singapore, 2000.

[13] H. Bao, J. H. Park, and J. Cao, "Synchronization of fractionalorder complex-valued neural networks with time delay," Neural Networks, vol. 81, pp. 16-28, 2016.

[14] L. Geng, Y. Yu, and S. Zhang, "Function projective synchronization between integer-order and stochastic fractional-order nonlinear systems," ISA Transactions ${ }^{\circledR}$, vol. 64, pp. 34-46, 2016.

[15] A. J. Muñoz-Vázquez, V. Parra-Vega, and A. Sánchez-Orta, "Continuous fractional-order sliding PI control for nonlinear systems subject to non-differentiable disturbances," Asian Journal of Control, vol. 19, no. 1, pp. 279-288, 2017.

[16] A. A. Khamzin, R. R. Nigmatullin, and I. . Popov, "Justification of the empirical laws of the anomalous dielectric relaxation in the framework of the memory function formalism," Fractional Calculus and Applied Analysis An International Journal for Theory and Applications, vol. 17, no. 1, pp. 247-258, 2014.

[17] C. W. Granger and R. Joyeux, "An introduction to long-memory time series models and fractional differencing," Journal of Time Series Analysis, vol. 1, no. 1, pp. 15-29, 1980.

[18] S. Bhalekar, V. Daftardar-Gejji, D. Baleanu, and R. Magin, "Transient chaos in fractional Bloch equations," Computers \&
Mathematics with Applications, vol. 64, no. 10, pp. 3367-3376, 2012.

[19] A. Elsonbaty, S. F. Hegazy, and S. S. A. Obayya, "Simultaneous Suppression of Time-Delay Signature in Intensity and Phase of Dual-Channel Chaos Communication," IEEE Journal of Quantum Electronics, vol. 51, no. 9, 2015.

[20] A. A. Elsadany and A. E. Matouk, "Dynamical behaviors of fractional-order Lotka-Volterra predator-prey model and its discretization," Applied Mathematics and Computation, vol. 49, no. 1-2, pp. 269-283, 2015.

[21] A. E. Matouk and A. A. Elsadany, "Dynamical analysis, stabilization and discretization of a chaotic fractional-order GLV model," Nonlinear Dynamics, vol. 85, no. 3, pp. 1597-1612, 2016.

[22] A. Elsadany, A. Matouk, A. Abdelwahab, and H. Abdallah, "Dynamical analysis, linear feedback control and synchronization of a generalized Lotka-Volterra system," in International Journal of Dynamics and Control, vol. 111, pp. 1-11, 2017.

[23] Y. Pan and H. Yu, "Biomimetic hybrid feedback feedforward neural-network learning control," IEEE Transactions on Neural Networks and Learning Systems, vol. 28, no. 6, pp. 1481-1487, 2017.

[24] A. Sambas, S. Vaidyanathan, M. Mamat, W. S. M. Sanjaya, and D. S. Rahayu, "A 3-D novel jerk chaotic system and its application in secure communication system and mobile robot navigation," Studies in Computational Intelligence, vol. 636, pp. 283-310, 2016.

[25] S. Vaidyanathan, C. K. Volos, V.-T. Pham, and A. T. Azar, "Hyperchaos, control, synchronization and circuit simulation of a novel 4-d hyperchaotic system with three quadratic nonlinearities," Studies in Fuzziness and Soft Computing, vol. 337, pp. 297-325, 2016.

[26] Z. Cheng, D. Li, and J. Cao, "Stability and Hopf bifurcation of a three-layer neural network model with delays," Neurocomputing, vol. 175, pp. 355-370, 2015.

[27] A. Boulkroune, "A fuzzy adaptive control approach for nonlinear systems with unknown control gain sign," Neurocomputing, vol. 179, pp. 318-325, 2016.

[28] Y. Pan, T. Sun, and H. Yu, "Peaking-free output-feedback adaptive neural control under a nonseparation principle," IEEE Transactions on Neural Networks and Learning Systems, vol. 26, no. 12, pp. 3097-3108, 2015.

[29] G. Velmurugan, R. Rakkiyappan, and J. Cao, "Finite-time synchronization of fractional-order memristor-based neural networks with time delays," Neural Networks, vol. 73, pp. 36-46, 2016.

[30] N. Bounar, A. Boulkroune, F. Boudjema, M. M'Saad, and M. Farza, "Adaptive fuzzy vector control for a doubly-fed induction motor," Neurocomputing, vol. 151, no. 2, pp. 756-769, 2015.

[31] Y. Pan, H. Yu, and M. J. Er, "Adaptive neural PD control with semiglobal asymptotic stabilization guarantee," IEEE Transactions on Neural Networks and Learning Systems, vol. 25, no. 12, pp. 2264-2274, 2014.

[32] C. Yin, S.-m. Zhong, and W.-f. Chen, "Design of sliding mode controller for a class of fractional-order chaotic systems," Communications in Nonlinear Science and Numerical Simulation, vol. 17, no. 1, pp. 356-366, 2012.

[33] A. Boulkroune, M. Tadjine, M. M'Saad, and M. Farza, "A unified approach for design of indirect adaptive output-feedback fuzzy controller," International Journal of Intelligent Systems Technologies and Applications, vol. 5, no. 1-2, pp. 83-103, 2008.

[34] W. S. Leaney, Methods and systems for processing microseismic data, 2016. 
[35] N. Kapoor, A. R. Teel, and P. Daoutidis, "An anti-windup design for linear systems with input saturation," Automatica, vol. 34, no. 5, pp. 559-574, 1998.

[36] K. Yakoubi and Y. Chitour, "Linear systems subject to input saturation and time delay: global asymptotic stabilization," Institute of Electrical and Electronics Engineers Transactions on Automatic Control, vol. 52, no. 5, pp. 874-879, 2007.

[37] G. Cui, S. Xu, F. L. Lewis, B. Zhang, and Q. Ma, "Distributed consensus tracking for non-linear multi-agent systems with input saturation: a command filtered backstepping approach," IET Control Theory \& Applications, vol. 10, no. 5, pp. 509-516, 2016.

[38] C. Li and W. Deng, "Remarks on fractional derivatives," Applied Mathematics and Computation, vol. 187, no. 2, pp. 777-784, 2007.

[39] Y. Li and S. Tong, "Adaptive Fuzzy Output-Feedback Control for Switched Nonlinear Systems with Arbitrary Switchings," Circuits, Systems and Signal Processing, vol. 35, no. 9, pp. 31523171, 2016.

[40] Y.-J. Liu, S. Tong, D.-J. Li, and Y. Gao, "Fuzzy adaptive control with state observer for a class of nonlinear discrete-time systems with input constraint," IEEE Transactions on Fuzzy Systems, vol. 24, no. 5, pp. 1147-1158, 2015.

[41] Y.-J. Liu, Y. Gao, S. Tong, and Y. Li, "Fuzzy approximation-based adaptive backstepping optimal control for a class of nonlinear discrete-time systems with dead-zone," IEEE Transactions on Fuzzy Systems, vol. 24, no. 1, pp. 16-28, 2016.

[42] Y. M. Li, S. C. Tong, and T. S. Li, "Hybrid fuzzy adaptive output feedback control design for uncertain MIMO nonlinear systems with time-varying delays and input saturation," IEEE Transactions on Fuzzy Systems, vol. 24, no. 4, pp. 841-853, 2015.

[43] H. Y. Li, C. W. Wu, L. G. Wu, H. K. Lam, and Y. B. Gao, "Filtering of interval type-2 fuzzy systems with intermittent measurements," IEEE Transactions on Cybernetics, vol. 46, no. 3, pp. 668-678, 2015.

[44] H. Li, C. W. Wu, S. Yin, and H. K. Lam, "Observer-based fuzzy control for nonlinear networked systems under unmeasurable premise variables," IEEE Transactions on Fuzzy Systems, vol. 24, no. 5, pp. 1233-1245, 2016.

[45] X. Yin, D. Yue, and S. Hu, "Adaptive periodic event-triggered consensus for multi-agent systems subject to input saturation," International Journal of Control, vol. 89, no. 4, pp. 653-667, 2016.

[46] M. K. Talkhoncheh, M. Shahrokhi, and M. R. Askari, "Observer-Based adaptive neural network controller for uncertain nonlinear systems with unknown control directions subject to input time delay and saturation," Information Sciences, vol. 418-419, pp. 717-737, 2017.

[47] H. Liu, S. Li, H. Wang, Y. Huo, and J. Luo, "Adaptive synchronization for a class of uncertain fractional-order neural networks," Entropy. An International and Interdisciplinary Journal of Entropy and Information Studies, vol. 17, no. 10, pp. 7185-7200, 2015.

[48] Y. Li, Y. Chen, and I. Podlubny, "Mittag-Leffler stability of fractional order nonlinear dynamic systems," Automatica, vol. 45, no. 8, pp. 1965-1969, 2009.

[49] J. G. Lu, "Chaotic dynamics and synchronization of fractionalorder Arneodos systems," Chaos, Solitons \& Fractals, vol. 26, no. 4, pp. 1125-1133, 2005.

[50] X. Y. Wang and J. M. Song, "Synchronization of the fractional order hyperchaos Lorenz systems with activation feedback control," Communications in Nonlinear Science and Numerical Simulation, vol. 14, no. 8, pp. 3351-3357, 2009. 




Advances in

Operations Research

vatersals



\section{The Scientific} World Journal

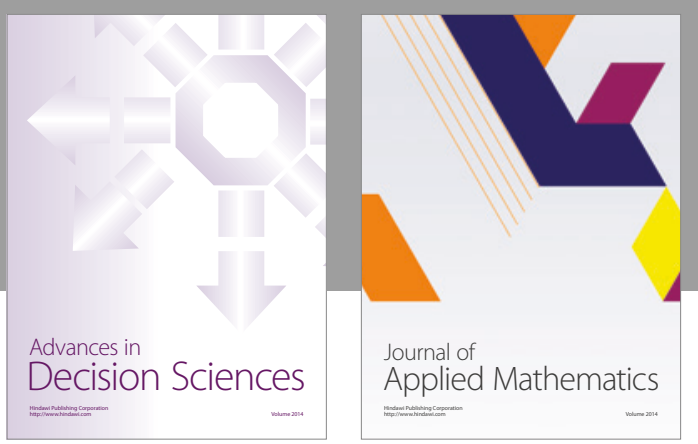

Algebra

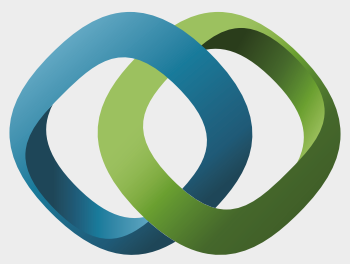

\section{Hindawi}

Submit your manuscripts at

https://www.hindawi.com
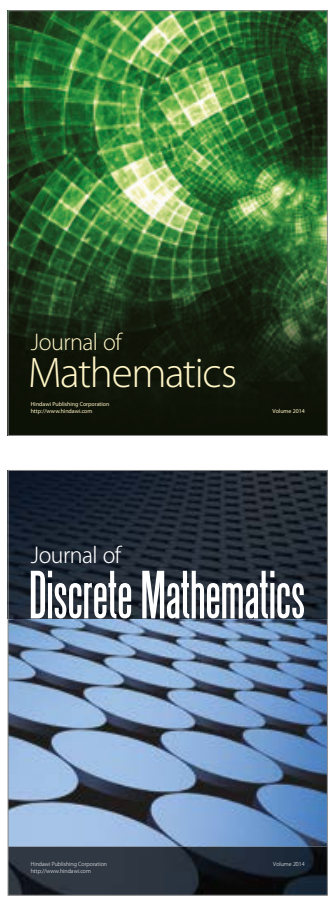

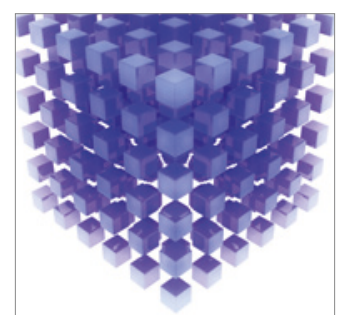

Mathematical Problems in Engineering
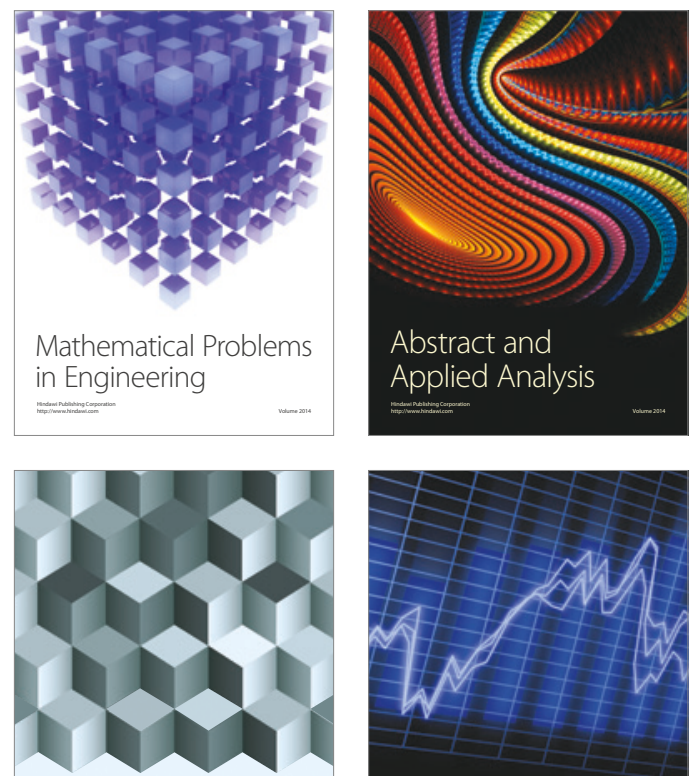

Journal of

Function Spaces



Probability and Statistics
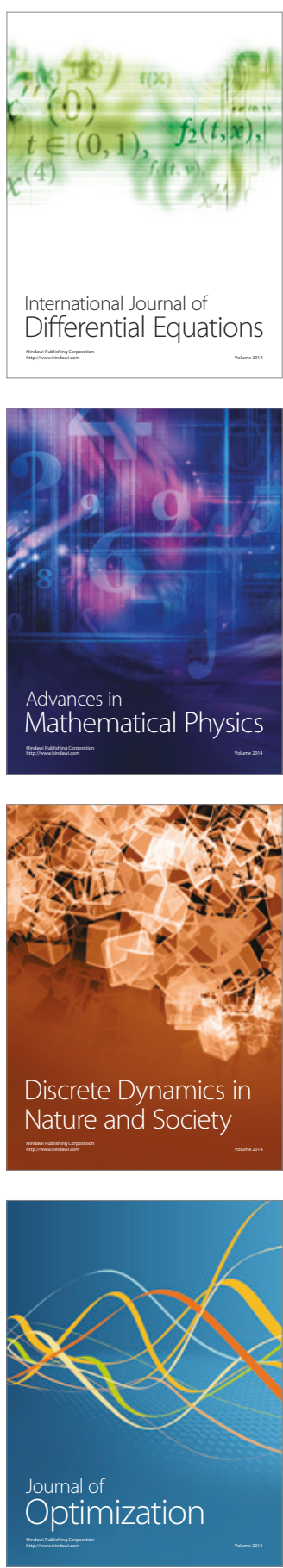